\title{
Non-perturbative approach to the attractive Hubbard model
}

\author{
S. Allen, and A.-M.S. Tremblay ${ }^{1}$ \\ Département de Physique and Centre de recherche sur les \\ propriétés électroniques de matériaux avancés. \\ ${ }^{1}$ Institut canadien de recherches avancées \\ Université de Sherbrooke, Sherbrooke, Québec, Canada J1K 2R1.
}

(November 5, 2018)

\begin{abstract}
A non-perturbative approach to the single-band attractive Hubbard model is presented in the general context of functional derivative approaches to many-body theories. As in previous work on the repulsive model, the first step is based on a local-field type ansatz, on enforcement of the Pauli principle and a number of crucial sum-rules. The Mermin-Wagner theorem in two dimensions is automatically satisfied. At this level, two-particle self-consistency has been achieved. In the second step of the approximation, an improved expression for the self-energy is obtained by using the results of the first step in an exact expression for the self-energy where the high- and low-frequency behaviors appear separately. The result is a cooperon-like formula. The required vertex corrections are included in this self-energy expression, as required by the absence of a Migdal theorem for this problem. Other approaches to the attractive Hubbard model are critically compared. Physical consequences of the present approach and agreement with Monte Carlo simulations are demonstrated in the accompanying paper (following this one).

PACS numbers: 71.10.Fd, 71.27.+a, 71.10.-w, 05.30.Fk.
\end{abstract}

\section{INTRODUCTION}

In the mid to late 1950's, quantum-field theoretical methods that had been developed first in the context of quantum electrodynamics began to have widespread applications in Condensed Matter Physics. [1,2] One can, roughly speaking, distinguish two types of approaches. The diagrammatic methods of Feynman and the functional methods of the Schwinger school $[3,4]$. Both points of view on many-body theory are equivalent. In particular, perturbation theory can be formulated diagrammatically or with functional methods. The two approaches in fact complement each other. For example, in calculating response functions, subsets of diagrams are often summed to infinite order. But naive resummations will generally break gauge invariance or other exact symmetry properties unless consistency between self-energy and two-particle irreducible vertices is enforced following a technique whose most natural formulation employs functional derivatives $[4,5]$.

Diagrammatic methods have nevertheless become by far the most popular techniques for many-body [6] problems in Condensed Matter, but the quest for non-perturbative approaches leads in general outside the realm of diagrams. While the Hartree-Fock approximation has a diagrammatic interpretation, what seems to be the most accurate approach to the electron gas at metallic densities (local field approximation (LFA) [7]) does not have a simple diagrammatic interpretation. In the case of the Hartree-Fock approximation, a variational principle guides the accuracy of the approximation. In the LFA, it is a self-consistency requirement at the two particle level that controls the accuracy.

Non-perturbative approaches have been developed in particular for the Hubbard model [8], perhaps the best know model for strongly interacting electrons on a lattice. While the early Green function decoupling schemes have largely fallen in disfavor because of their ad hoc and uncontrolled nature, many successful non-perturbative approaches have been developed in one dimension. Recently, dynamical mean-field theory has become the method of choice [9] for higher dimensions. This method, however, is in fact based on an expansion about infinite dimension. In two dimensions in particular, the momentum dependence of the self-energy cannot be neglected and this method becomes less accurate.

The purpose of this paper is to extend to the attractive Hubbard model a non-perturbative approach developed previously for the repulsive model [10-12]. This method was an extension of the LFA work of Singwi, Tosi, Land and Sjölander [7] for the electron gas and of Hedeyati and Vignale for the Hubbard model [13]. It went further than these work in imposing the Pauli principle, a number of exact sum rules, of conservation laws, and proposing a formula for the self-energy in the paramagnetic state that includes momentum and frequency dependence. The approach also includes an internal check on accuracy based on an exact relationship between one and two-particle properties. Although the method fails in strong coupling or very close to a critical point, it gives the most accurate results 
when compared with Monte Carlo simulations in the weak to intermediate coupling regime [12]. The present paper generalizes that approach to the attractive Hubbard model. The accompanying paper [14] demonstrates accuracy by comparisons with Monte Carlo simulations, and discusses a problem of importance in the context of high-temperature [15] and organic [16] superconductors, namely the opening of a pairing-fluctuation induced pseudogap.

The structure of this paper is as follows. In section II we introduce the general many-body formalism to obtain expressions for the self-energy and irreducible vertices in the functional derivative approach. Although in principle standard, the functional derivative approach in the particle-particle channel is not widely used. It allows us to establish a number of exact results. At this level, all the results could have been obtained with formal diagrammatic expansions in skeleton diagrams, but we refrain from doing this since the functional approach is far more economical in this context. The exact results that we present form the basis of the Two-Particle Self-Consistent (TPSC) approach and its extension (section III). These are non-perturbative approximations that are not diagrammatic resummations. A discussion of the various exact results that our approach satisfies either exactly or self-consistently is then presented in section IV. Details of the derivation of the exact results, namely sum rules and high-frequency expansions, are given in Appendix A. The last appendix compares our approach with various other approaches and explains the connection with the formalism for the repulsive Hubbard model. A summary of our main results is in the conclusion, section $\mathrm{V}$.

\section{EXACT RELATIONSHIPS BETWEEN GREEN FUNCTION, SELF-ENERGY AND VERTICES}

In this section, we derive a number of exact results using the functional derivative formalism. It is on the basis of these results, and using again the functional derivative approach, that our approximation scheme will be developed in section III.

\section{A. Definitions, equations of motion}

We work with creation-annihilation operators $\psi_{\uparrow}^{\dagger}, \psi_{\downarrow}$ for Wannier states of spin $\sigma=\uparrow, \downarrow$ located at position $\mathbf{r}_{1}$, and, in the Heisenberg representation, imaginary time $\tau_{1}$. The space and imaginary time indices are abbreviated by arabic numerals. Furthermore, we use the Nambu representation,

$$
\begin{aligned}
\Psi^{\dagger}(1) & =\left(\psi_{\uparrow}^{\dagger}(1), \psi_{\downarrow}(1)\right) \\
\Psi(1) & =\left(\begin{array}{c}
\psi_{\uparrow}(1) \\
\psi_{\downarrow}^{\dagger}(1)
\end{array}\right)
\end{aligned}
$$

where the field operators obey the anticommutation relations

$$
\left\{\Psi_{\alpha}(1), \Psi_{\beta}^{\dagger}(2)\right\} \delta\left(\tau_{1}-\tau_{2}\right)=\delta(1-2) \delta_{\alpha, \beta} .
$$

In this notation, the space part of Dirac delta functions are Kroenecker deltas: $\delta(1-2)=\delta_{\mathbf{r}_{1}, \mathbf{r}_{2}} \delta\left(\tau_{1}-\tau_{2}\right)$. When numerals are set in bold face, we mean just the space position (e.g. $t(\mathbf{1}, \mathbf{2}))$ and refer to the Schrödinger picture. Adding the convention that indices with an overbar are summed over space positions and, when appropriate, integrated over imaginary time from 0 to $\beta$, the Hubbard Hamiltonian takes the form

$$
\widehat{H}=-t(\overline{\mathbf{1}}, \overline{\mathbf{2}})\left(\psi_{\bar{\sigma}}^{\dagger}(\overline{\mathbf{1}}) \psi_{\bar{\sigma}}(\overline{\mathbf{2}})+\psi_{\bar{\sigma}}^{\dagger}(\overline{\mathbf{2}}) \psi_{\bar{\sigma}}(\overline{\mathbf{1}})\right)+U \psi_{\uparrow}^{\dagger}(\overline{\mathbf{1}}) \psi_{\uparrow}(\overline{\mathbf{1}}) \psi_{\downarrow}^{\dagger}(\overline{\mathbf{1}}) \psi_{\downarrow}(\overline{\mathbf{1}})
$$

with $t(\overline{\mathbf{1}}, \overline{\mathbf{2}})$ the hopping matrix elements.

Since we will work in the grand-canonical ensemble, it is convenient to take $\widehat{H}-\mu \widehat{N}$ as the time evolution operator in the Heisenberg representation, with $\mu$ the chemical potential and $\widehat{N}$ the number operator. The corresponding equations of motion for the field operators then are,

$$
\begin{aligned}
& {\left[\left(\frac{\partial}{\partial \tau_{1}}-\mu\right) \delta_{\mathbf{r}_{1}, \overline{\mathbf{r}}}-t(\mathbf{1}, \overline{\mathbf{2}})\right] \delta\left(\tau_{1}-\tau_{\overline{2}}\right) \psi_{\sigma}(\overline{2})=-U \psi_{-\sigma}^{\dagger}(1) \psi_{-\sigma}(1) \psi_{\sigma}(1)} \\
& {\left[\left(\frac{\partial}{\partial \tau_{1}}+\mu\right) \delta_{\mathbf{r}_{1}, \mathbf{r}_{\overline{2}}}+t(\mathbf{1}, \overline{\mathbf{2}})\right] \delta\left(\tau_{1}-\tau_{\overline{2}}\right) \psi_{\sigma}^{\dagger}(\overline{2})=U \psi_{-\sigma}^{\dagger}(1) \psi_{-\sigma}(1) \psi_{\sigma}^{\dagger}(1)}
\end{aligned}
$$


We will also need the momentum space representation

$$
\psi_{\sigma}(\mathbf{1})=\frac{1}{\sqrt{N}} \sum_{\mathbf{k}} e^{i \mathbf{k} \cdot \mathbf{r}_{1}} c_{\mathbf{k}, \sigma} ; \quad \psi_{\sigma}^{\dagger}(\mathbf{1})=\frac{1}{\sqrt{N}} \sum_{\mathbf{k}} e^{-i \mathbf{k} \cdot \mathbf{r}_{1}} c_{\mathbf{k}, \sigma}^{\dagger}
$$

and the pair operators

$$
\Delta(\mathbf{1})=\psi_{\uparrow}(\mathbf{1}) \psi_{\downarrow}(\mathbf{1}) \quad ; \quad \Delta^{\dagger}(\mathbf{1})=\psi_{\downarrow}^{\dagger}(\mathbf{1}) \psi_{\uparrow}^{\dagger}(\mathbf{1})
$$

with Fourier transform

$$
\Delta_{\mathbf{q}}=\frac{1}{\sqrt{N}} \sum_{\mathbf{k}} c_{\mathbf{k}, \uparrow} c_{-\mathbf{k}+\mathbf{q}, \downarrow} ; \quad \Delta_{\mathbf{q}}^{\dagger}=\frac{1}{\sqrt{N}} \sum_{\mathbf{k}} c_{-\mathbf{k}+\mathbf{q}, \downarrow}^{\dagger} c_{\mathbf{k}, \uparrow}^{\dagger}
$$

These pair operators do not include the interaction potential in their definition. The pair operators obey the equations of motion,

$$
\begin{aligned}
& \frac{\partial \Delta_{\mathbf{q}}}{\partial \tau}=\frac{-1}{\sqrt{N}} \sum_{\mathbf{k}}\left[\varepsilon_{\mathbf{k}}+\varepsilon_{-\mathbf{k}+\mathbf{q}}-2\left(\mu-\frac{U}{2}\right)\right] c_{\mathbf{k}, \uparrow} c_{-\mathbf{k}+\mathbf{q}, \downarrow} \\
& \frac{\partial \Delta_{\mathbf{q}}^{\dagger}}{\partial \tau}=\frac{1}{\sqrt{N}} \sum_{\mathbf{k}}\left[\varepsilon_{\mathbf{k}}+\varepsilon_{-\mathbf{k}+\mathbf{q}}-2\left(\mu-\frac{U}{2}\right)\right] c_{-\mathbf{k}+\mathbf{q}, \downarrow}^{\dagger} c_{\mathbf{k}, \uparrow}^{\dagger}
\end{aligned}
$$

where the band dispersion $\varepsilon_{\mathbf{k}}$ is the Fourier transform of the hopping matrix elements

$$
-t(\mathbf{1}, \mathbf{2})=\frac{1}{N} \sum_{\mathbf{k}} e^{i \mathbf{k} \cdot\left(\mathbf{r}_{1}-\mathbf{r}_{2}\right)} \varepsilon_{\mathbf{k}}
$$

\section{B. Green function and self-energy}

As in Ref. [3], we work in the grand canonical ensemble in the presence of auxiliary source fields that are useful in intermediate steps of the calculations. The source fields are set to zero at the end. More specifically, we define the expectation value of a general time-ordered operator $O$ by

$$
\left\langle T_{\tau}[O]\right\rangle_{\Theta} \equiv Z^{-1}(\{\boldsymbol{\Theta}\}) \operatorname{Tr}\left\{e^{-\beta(\widehat{H}-\mu \widehat{N})} T_{\tau}\left[e^{-\Psi^{\dagger}(\overline{1}) \Theta(\overline{1}, \overline{2}) \Psi(\overline{2})} O\right]\right\}
$$

with

$$
Z(\{\boldsymbol{\Theta}\}) \equiv \operatorname{Tr}\left\{e^{-\beta(\widehat{H}-\mu \widehat{N})} T_{\tau}\left[e^{-\Psi^{\dagger}(\overline{1}) \Theta(\overline{1}, \overline{2}) \Psi(\overline{2})}\right]\right\}
$$

where $T_{\tau}$ is the time-ordering operator while the matrix source field

$$
\Theta(1,2)=\left(\begin{array}{cc}
0 & \theta(1,2) \\
\theta^{*}(1,2) & 0
\end{array}\right)
$$

physically corresponds to Cooper pair sources,

$$
\Psi^{\dagger}(\overline{1}) \Theta(\overline{1}, \overline{2}) \Psi(\overline{2})=\theta(\overline{1}, \overline{2}) \psi_{\uparrow}^{\dagger}(\overline{1}) \psi_{\downarrow}^{\dagger}(\overline{2})+\theta^{*}(\overline{1}, \overline{2}) \psi_{\downarrow}(\overline{1}) \psi_{\uparrow}(\overline{2}) .
$$

The Nambu Green's function is then a functional of the auxiliary field $\Theta$ defined by

$$
\mathbf{G}(1,2 ;\{\boldsymbol{\Theta}\}) \equiv-\left\langle T_{\tau}\left[\Psi(1) \Psi^{\dagger}(2)\right]\right\rangle_{\boldsymbol{\Theta}}=-\left(\begin{array}{cc}
\left\langle T_{\tau}\left[\psi_{\uparrow}(1) \psi_{\uparrow}^{\dagger}(2)\right]\right\rangle_{\boldsymbol{\Theta}} & \left\langle T_{\tau}\left[\psi_{\uparrow}(1) \psi_{\downarrow}(2)\right]\right\rangle_{\boldsymbol{\Theta}} \\
\left\langle T_{\tau}\left[\psi_{\downarrow}^{\dagger}(1) \psi_{\uparrow}^{\dagger}(2)\right]\right\rangle_{\boldsymbol{\Theta}} & \left\langle T_{\tau}\left[\psi_{\downarrow}^{\dagger}(1) \psi_{\downarrow}(2)\right]\right\rangle_{\boldsymbol{\Theta}}
\end{array}\right)
$$


The quantities that will appear at the end of the calculation will, in analogy with the Green function, be defined in zero source field, namely

$$
\mathbf{G}(1,2) \equiv \mathbf{G}(1,2 ; \mathbf{0})=-\left\langle T_{\tau}\left[\Psi(1) \Psi^{\dagger}(2)\right]\right\rangle
$$

Using the equations of motion (5) and following the algebra of Ref. [17], one finds that

$$
\mathbf{G}^{-1}(1,2 ;\{\boldsymbol{\Theta}\})=\mathbf{G}_{0}^{-1}(1,2)-\boldsymbol{\Sigma}(1,2 ;\{\boldsymbol{\Theta}\})-\boldsymbol{\Theta}(1,2)
$$

where in Matsubara frequency $\left(k_{n}=(2 n+1) \pi T\right)$ and Fourier space $(\mathbf{k})$, the non-interacting Green function takes its usual form,

$$
\mathbf{G}_{0}\left(\mathbf{k}, i k_{n}\right)=\left(\begin{array}{cc}
\frac{1}{i k_{n}-\left(\varepsilon_{\mathbf{k}}-\mu\right)} & 0 \\
0 & \frac{-1}{-i k_{n}-\left(\varepsilon_{-\mathbf{k}}-\mu\right)}
\end{array}\right)
$$

while the effects of interactions are contained in the self-energy matrix defined by

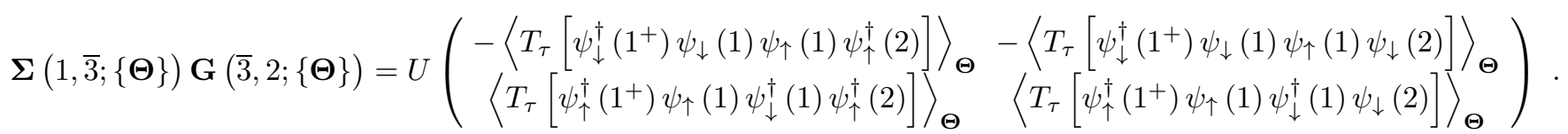

The notation $1^{+}$indicates that the imaginary time is infinitesimally larger than $\tau_{1}$, (or smaller for $1^{-}$) .

\section{Self-energy and pair susceptibility}

The self-energy, as should be clear from the last equation, depends on four-point functions that may be calculated in different channels. For the repulsive Hubbard model, charge and spin fluctuation channels are dominant, so approximations for the four point functions are written down in these channels. However, in the nearest-neighbor attractive model away from half-filling, it is the pair fluctuations that are dominant, even in the paramagnetic state. As a preliminary remark, we can suggest how the self-energy will be related to the pair correlation function by making the key observation that the diagonal terms can be written as functional derivatives with respect to the $\theta$ field. First, note that when $\theta$ is set to zero in Eq.(21) it becomes diagonal and $\boldsymbol{\Sigma}_{11}$ simplifies to

$$
\boldsymbol{\Sigma}_{11}(1,2)=-U\left\langle T_{\tau}\left[\psi_{\downarrow}^{\dagger}\left(1^{+}\right) \psi_{\downarrow}(1) \psi_{\uparrow}(1) \psi_{\uparrow}^{\dagger}(\overline{3})\right]\right\rangle G_{11}^{-1}(\overline{3}, 2) .
$$

The operators located in the middle, $\psi_{\downarrow}(1) \psi_{\uparrow}(1)$, can be obtained from a functional derivative with respect to $\theta^{*}(1,1)$ before this field is set to zero. Hence, taking the functional derivative and setting off-diagonal terms to zero afterwards, one is left with

$$
\boldsymbol{\Sigma}_{11}(1,2)=-\left.U \frac{\delta G_{21}\left(1^{+}, \overline{3} ;\{\boldsymbol{\Theta}\}\right)}{\delta \theta^{*}(1,1)}\right|_{\Theta=0} G_{11}^{-1}(\overline{3}, 2)
$$

This is basically what we want. The self-energy will be written in terms of a response function in the particle-particle channel.

To continue more generally, we step back and define the susceptibility matrix

$$
\mathbf{X}(1,2,3,4 ;\{\boldsymbol{\Theta}\})=-\frac{\delta}{\delta \boldsymbol{\Theta}(4,3)} \mathbf{G}(1,2 ;\{\boldsymbol{\Theta}\})
$$

where $\delta / \delta \Theta(3,4)$ is a matrix operator in Nambu space

$$
\frac{\delta}{\delta \Theta(3,4)}=\left(\begin{array}{cc}
0 & \frac{\delta}{\delta \theta^{*}(3,4)} \\
\frac{\delta}{\delta \theta(3,4)} & 0
\end{array}\right)
$$

such that 


$$
\frac{\delta}{\delta \Theta(3,4)} \boldsymbol{\Theta}(1,2)=\delta(1-3) \delta(2-4) \mathbf{I}
$$

where $\mathbf{I}$ is the identity matrix in Nambu space. Note that the susceptibility $\mathbf{X}$ is still a matrix in Nambu space with only two matrix indices (four matrix elements). With this notation, the two-point function that we will need to compute the self-energy from Eq.(21) is the following special case of Eq.(24): $\mathbf{X}\left(1,2,1^{\mp}, 1^{\mp} ;\{\boldsymbol{\Theta}\}\right)$. Evaluating the functional differentiation explicitly, we have

$$
\begin{aligned}
\mathbf{X}\left(1,2,1^{\mp}, 1^{\mp} ;\{\boldsymbol{\Theta}\}\right) \equiv & -\left(\begin{array}{cc}
0 & \frac{\delta}{\delta \theta^{*}\left(1^{-}, 1^{-}\right)} \\
\frac{\delta}{\delta \theta\left(1^{+}, 1^{+}\right)} & 0
\end{array}\right)\left(\begin{array}{cc}
-\left\langle T_{\tau}\left[\psi_{\uparrow}(1) \psi_{\uparrow}^{\dagger}(2)\right]\right\rangle_{\Theta} & -\left\langle T_{\tau}\left[\psi_{\uparrow}(1) \psi_{\downarrow}(2)\right]\right\rangle_{\Theta} \\
-\left\langle T_{\tau}\left[\psi_{\downarrow}^{\dagger}(1) \psi_{\uparrow}^{\dagger}(2)\right]\right\rangle_{\Theta}-\left\langle T_{\tau}\left[\psi_{\downarrow}^{\dagger}(1) \psi_{\downarrow}(2)\right]\right\rangle_{\Theta}
\end{array}\right) \\
= & \left(\begin{array}{cc}
\frac{\delta}{\delta \theta^{*}\left(1^{-}, 1^{-}\right)}\left\langle T_{\tau}\left[\psi_{\downarrow}^{\dagger}(1) \psi_{\uparrow}^{\dagger}(2)\right]\right\rangle_{\boldsymbol{\Theta}} \frac{\delta}{\delta \theta^{*}\left(1^{-}, 1^{-}\right)}\left\langle T_{\tau}\left[\psi_{\downarrow}^{\dagger}(1) \psi_{\downarrow}(2)\right]\right\rangle_{\Theta} \\
\frac{\delta}{\delta \theta\left(1^{+}, 1^{+}\right)}\left\langle T_{\tau}\left[\psi_{\uparrow}(1) \psi_{\uparrow}^{\dagger}(2)\right]\right\rangle_{\Theta} & \frac{\delta}{\delta \theta\left(1^{+}, 1^{+}\right)}\left\langle T_{\tau}\left[\psi_{\uparrow}(1) \psi_{\downarrow}(2)\right]\right\rangle_{\Theta}
\end{array}\right) \\
= & -\left(\begin{array}{l}
\left\langle T_{\tau}\left[\psi_{\downarrow}^{\dagger}(1) \psi_{\downarrow}\left(1^{-}\right) \psi_{\uparrow}\left(1^{-}\right) \psi_{\uparrow}^{\dagger}(2)\right]\right\rangle_{\boldsymbol{\Theta}}\left\langle T_{\tau}\left[\psi_{\downarrow}^{\dagger}(1) \psi_{\downarrow}\left(1^{-}\right) \psi_{\uparrow}\left(1^{-}\right) \psi_{\downarrow}(2)\right]\right\rangle_{\Theta} \\
\left\langle T_{\tau}\left[\psi_{\uparrow}(1) \psi_{\uparrow}^{\dagger}\left(1^{+}\right) \psi_{\downarrow}^{\dagger}\left(1^{+}\right) \psi_{\uparrow}^{\dagger}(2)\right]\right\rangle_{\Theta}\left\langle T_{\tau}\left[\psi_{\uparrow}(1) \psi_{\uparrow}^{\dagger}\left(1^{+}\right) \psi_{\downarrow}^{\dagger}\left(1^{+}\right) \psi_{\downarrow}(2)\right]\right\rangle_{\Theta}
\end{array}\right) \\
& -\mathbf{F}\left(1^{\mp}, 1^{\mp} ;\{\boldsymbol{\Theta}\}\right) \mathbf{G}(1,2 ;\{\boldsymbol{\Theta}\})
\end{aligned}
$$

where we defined a function $\mathbf{F}$ that contains only the anomalous pieces of the full Green function

$$
\mathbf{F}\left(1^{\mp}, 1^{\mp} ;\{\boldsymbol{\Theta}\}\right) \equiv\left(\begin{array}{cc}
0 & -\left\langle T_{\tau}\left[\psi_{\uparrow}\left(1^{-}\right) \psi_{\downarrow}\left(1^{-}\right)\right]\right\rangle_{\Theta} \\
-\left\langle T_{\tau}\left[\psi_{\downarrow}^{\dagger}\left(1^{+}\right) \psi_{\uparrow}^{\dagger}\left(1^{+}\right)\right]\right\rangle_{\Theta} & 0
\end{array}\right) .
$$

The last term in the above expression for the pair susceptibility comes from functional differentiation of the denominator in the definition of averages, as one can check with the definition Eq.(14) for the partition function,

$$
\left(\begin{array}{cc}
0 & \frac{\delta}{\delta \theta^{*}\left(1^{-}, 1^{-}\right)} \\
\frac{\delta}{\delta \theta\left(1^{+}, 1^{+}\right)} & 0
\end{array}\right) \frac{1}{Z(\{\boldsymbol{\Theta}\})}=\left(\begin{array}{cc}
0 & -\left\langle T_{\tau}\left[\psi_{\downarrow}\left(1^{-}\right) \psi_{\uparrow}\left(1^{-}\right)\right]\right\rangle_{\Theta} \\
-\left\langle T_{\tau}\left[\psi_{\uparrow}^{\dagger}\left(1^{+}\right) \psi_{\downarrow}^{\dagger}\left(1^{+}\right)\right]\right\rangle_{\Theta} & 0
\end{array}\right) \frac{-1}{Z(\{\boldsymbol{\Theta}\})} .
$$

Using rotational invariance and our general result for the self-energy Eq.(21), we finally find the following key relation between self-energy and susceptibility

$$
\boldsymbol{\Sigma}(1, \overline{3} ;\{\boldsymbol{\Theta}\}) \mathbf{G}(\overline{3}, 2 ;\{\boldsymbol{\Theta}\})=U \mathbf{X}\left(1,2,1^{\mp}, 1^{\mp} ;\{\boldsymbol{\Theta}\}\right)+U \mathbf{F}\left(1^{\mp}, 1^{\mp} ;\{\boldsymbol{\Theta}\}\right) \mathbf{G}(1,2 ;\{\boldsymbol{\Theta}\})
$$

or

$$
\boldsymbol{\Sigma}(1,2 ;\{\boldsymbol{\Theta}\})=U \mathbf{X}\left(1, \overline{3}, 1^{\mp}, 1^{\mp} ;\{\boldsymbol{\Theta}\}\right) \mathbf{G}^{-1}(\overline{3}, 2 ;\{\boldsymbol{\Theta}\})+U \mathbf{F}\left(1^{\mp}, 1^{\mp} ;\{\boldsymbol{\Theta}\}\right) \delta(1-2) \mathbf{I}
$$

\section{Bethe-Salpeter equation for the three-point susceptibility and relationship between irreducible vertices and self-energy}

In this section, we derive the Bethe-Salpeter equation for the susceptibility using the functional derivative scheme [3]. This equation allows to define the two-particle irreducible vertex that plays for the susceptibility a role analogous to that of the self-energy for the Green function. In deriving the Bethe-Salpeter equation, we will recover the well known relation between self-energy and particle-particle irreducible vertices. Since the susceptibility is the functional derivative of $\mathbf{G}$ while the self-energy is trivially related to $\mathbf{G}^{-1}$, it is natural to start from

$$
\mathbf{G}(1, \overline{4} ;\{\boldsymbol{\Theta}\}) \mathbf{G}^{-1}(\overline{4}, 5 ;\{\boldsymbol{\Theta}\})=\delta(1-5) \mathbf{I}
$$

and to take the functional derivative of this equation. One finds,

$$
\frac{\delta}{\delta \boldsymbol{\Theta}(2,2)}\left[\mathbf{G}(1, \overline{4} ;\{\boldsymbol{\Theta}\}) \mathbf{G}^{-1}(\overline{4}, 5 ;\{\boldsymbol{\Theta}\})\right]=0
$$




$$
\begin{aligned}
{\left[\frac{\delta}{\delta \boldsymbol{\Theta}(2,2)} \mathbf{G}(1, \overline{4} ;\{\boldsymbol{\Theta}\})\right] \mathbf{G}^{-1}(\overline{4}, 5 ;\{\boldsymbol{\Theta}\})=} & -\sigma_{+} \mathbf{G}(1, \overline{4})\left(\frac{\delta}{\delta \theta^{*}(2,2)} \mathbf{G}^{-1}(\overline{4}, 5 ;\{\boldsymbol{\Theta}\})\right) \\
& -\sigma_{-} \mathbf{G}(1, \overline{4})\left(\frac{\delta}{\delta \theta(2,2)} \mathbf{G}^{-1}(\overline{4}, 5 ;\{\boldsymbol{\Theta}\})\right)
\end{aligned}
$$

where we used the definitions

$$
\sigma_{+} \equiv\left(\begin{array}{cc}
0 & 1 \\
0 & 0
\end{array}\right) \quad ; \quad \sigma_{-} \equiv\left(\begin{array}{cc}
0 & 0 \\
1 & 0
\end{array}\right)
$$

Using the expression for the inverse Green function Eq.(19) and the chain rule to take into account the dependence of $\boldsymbol{\Sigma}$ on $\{\boldsymbol{\Theta}\}$ through [18] $\mathbf{G}$, one obtains,

$$
\begin{aligned}
& \frac{\delta}{\delta \theta^{*}(2,2)} \mathbf{G}^{-1}(4,5 ;\{\boldsymbol{\Theta}\})=-\delta(2-5) \delta(2-4) \sigma_{-}-\frac{\delta \boldsymbol{\Sigma}(4,5 ;\{\boldsymbol{\Theta}\})}{\delta G_{\overline{k \ell}}(\overline{6}, \overline{7} ;\{\boldsymbol{\Theta}\})} \frac{\delta G_{\overline{k \ell}}(\overline{6}, \overline{7} ;\{\boldsymbol{\Theta}\})}{\delta \theta^{*}(2,2)} \\
& \frac{\delta}{\delta \theta(2,2)} \mathbf{G}^{-1}(4,5 ;\{\boldsymbol{\Theta}\})=-\delta(2-5) \delta(2-4) \sigma_{+}-\frac{\delta \boldsymbol{\Sigma}(4,5 ;\{\boldsymbol{\Theta}\})}{\delta G_{\overline{k \ell}}(\overline{6}, \overline{7} ;\{\boldsymbol{\Theta}\})} \frac{\delta G_{\overline{k \ell}}(\overline{6}, \overline{7} ;\{\boldsymbol{\Theta}\})}{\delta \theta(2,2)} .
\end{aligned}
$$

The above equations simplify greatly when the functional derivative is evaluated in the normal, zero source field case, where particle number conservation implies the vanishing of anomalous correlation functions. We are left with,

$$
\begin{gathered}
\left.\frac{\delta G_{\overline{k \ell}}(6,7 ;\{\boldsymbol{\Theta}\})}{\delta \theta^{*}(2,2)}\right|_{\boldsymbol{\Theta}=\mathbf{0}}=\left.\delta_{\bar{k}, 2} \delta_{\bar{\ell}, 1} \frac{\delta G_{21}(6,7 ;\{\boldsymbol{\Theta}\})}{\delta \theta^{*}(2,2)}\right|_{\boldsymbol{\Theta}=\mathbf{0}} \\
\left.\frac{\delta G_{\overline{k \ell}}(6,7 ;\{\boldsymbol{\Theta}\})}{\delta \theta(2,2)}\right|_{\boldsymbol{\Theta}=\mathbf{0}}=\left.\delta_{\bar{k}, 1} \delta_{\bar{\ell}, 2} \frac{\delta G_{12}(6,7 ;\{\boldsymbol{\Theta}\})}{\delta \theta(2,2)}\right|_{\boldsymbol{\Theta}=\mathbf{0}} .
\end{gathered}
$$

Using the latter results in Eq.(37) multiplying it by $\mathbf{G}(5,3)$ and then integrating over the point 5 , we find, with the help of Eqs.(39) and (40),

$$
\begin{aligned}
\left.\frac{\delta}{\delta \boldsymbol{\Theta}(2,2)} \mathbf{G}(1,3 ;\{\boldsymbol{\Theta}\})\right|_{\boldsymbol{\Theta}=\mathbf{0}}= & \sigma_{+} \mathbf{G}(1,2) \sigma_{-} \mathbf{G}(2,3)+\sigma_{-} \mathbf{G}(1,2) \sigma_{+} \mathbf{G}(2,3) \\
& +\left.\left.\sigma_{+} \mathbf{G}(1, \overline{4}) \frac{\delta \boldsymbol{\Sigma}(\overline{4}, \overline{5} ;\{\boldsymbol{\Theta}\})}{\delta G_{21}(\overline{6}, \overline{7})}\right|_{\boldsymbol{\Theta}=\mathbf{0}} \frac{\delta G_{21}(\overline{6}, \overline{7} ;\{\boldsymbol{\Theta}\})}{\delta \theta^{*}(2,2)}\right|_{\boldsymbol{\Theta}=\mathbf{0}} \mathbf{G}(\overline{5}, 3) \\
& +\left.\left.\sigma_{-} \mathbf{G}(1, \overline{4}) \frac{\delta \boldsymbol{\Sigma}(\overline{4}, \overline{5} ;\{\boldsymbol{\Theta}\})}{\delta G_{12}(\overline{6}, \overline{7})}\right|_{\boldsymbol{\Theta}=\mathbf{0}} \frac{\delta G_{12}(\overline{6}, \overline{7} ;\{\boldsymbol{\Theta}\})}{\delta \theta(2,2)}\right|_{\boldsymbol{\Theta}=\mathbf{0}} \mathbf{G}(\overline{5}, 3) .
\end{aligned}
$$

Since we consider the normal phase, the off-diagonal Green functions vanish and we are left with only two equations that come from the diagonal components of the above matrix equation. The off-diagonal parts just tell us that two of the irreducible vertices vanish. Finally then,

$$
\begin{gathered}
\left.\frac{\delta G_{21}(1,3 ;\{\boldsymbol{\Theta}\})}{\delta \theta^{*}(2,2)}\right|_{\boldsymbol{\Theta}=\mathbf{0}}=G_{22}(1,2) G_{11}(2,3)+\left.\left.G_{22}(1, \overline{4}) \frac{\delta \Sigma_{21}(\overline{4}, \overline{5} ;\{\boldsymbol{\Theta}\})}{\delta G_{21}(\overline{6}, \overline{7})}\right|_{\boldsymbol{\Theta}=\mathbf{0}} \frac{\delta G_{21}(\overline{6}, \overline{7} ;\{\boldsymbol{\Theta}\})}{\delta \theta^{*}(2,2)}\right|_{\boldsymbol{\Theta}=\mathbf{0}} G_{11}(\overline{5}, 3) \\
\left.\frac{\delta G_{12}(1,3 ;\{\boldsymbol{\Theta}\})}{\delta \theta(2,2)}\right|_{\boldsymbol{\Theta}=\mathbf{0}}=G_{11}(1,2) G_{22}(2,3)+\left.\left.G_{11}(1, \overline{4}) \frac{\delta \Sigma_{12}(\overline{4}, \overline{5} ;\{\boldsymbol{\Theta}\})}{\delta G_{12}(\overline{6}, \overline{7})}\right|_{\boldsymbol{\Theta}=\mathbf{0}} \frac{\delta G_{12}(\overline{6}, \overline{7} ;\{\boldsymbol{\Theta}\})}{\delta \theta(2,2)}\right|_{\boldsymbol{\Theta}=\mathbf{0}} G_{22}(\overline{5}, 3) .
\end{gathered}
$$




\section{E. An exact expression for the self-energy where low- and high-frequency behaviors are separated.}

The high-frequency limit of the self-energy is given by the Hartree-Fock result, as can be shown from sum rules. For latter purposes in our approximative scheme, it is useful to have at hand an exact expression for the self-energy where the high-frequency behavior appears explicitly. In this section, we derive such an expression in the case where the auxiliary field $\boldsymbol{\Theta}$ vanishes.

First, let us recall the sum rules that fix the high-frequency behavior of the self-energy. In the absence of external field

$$
\mathbf{G}\left(\mathbf{k}, i k_{n}\right)=\int \frac{d \omega}{2 \pi} \frac{\mathbf{A}(\mathbf{k}, \omega)}{i k_{n}-\omega}
$$

where the single-particle spectral weight $\mathbf{A}(\mathbf{k}, \omega)$ for the Nambu Green function is given by,

$$
\mathbf{A}(\mathbf{k}, \omega)=\int d t e^{i \omega t}\left(\begin{array}{cc}
\left\langle\left\{c_{\mathbf{k}, \uparrow}(t), c_{\mathbf{k}, \uparrow}^{\dagger}\right\}\right\rangle & 0 \\
0 & \left\langle\left\{c_{\mathbf{k}, \downarrow}^{\dagger}(t), c_{\mathbf{k}, \downarrow}\right\}\right\rangle
\end{array}\right) .
$$

The high-frequency expansion of the Green function is then

$$
\mathbf{G}\left(\mathbf{k}, i k_{n}\right)=\frac{1}{i k_{n}} \int \frac{d \omega}{2 \pi} \mathbf{A}(\mathbf{k}, \omega)+\frac{1}{\left(i k_{n}\right)^{2}} \int \frac{d \omega}{2 \pi} \omega \mathbf{A}(\mathbf{k}, \omega)+\ldots
$$

where the frequency moments of $\mathbf{A}(\mathbf{k}, \omega)$ are easily computed from equal-time commutators

$$
\begin{gathered}
\int \frac{d \omega}{2 \pi} \mathbf{A}(\mathbf{k}, \omega)=\mathbf{I} \\
\int \frac{d \omega}{2 \pi} \omega \mathbf{A}(\mathbf{k}, \omega)=\left(\varepsilon_{\mathbf{k}}-\mu\right) \sigma_{z}+U\left(\begin{array}{cc}
\left\langle n_{\downarrow}\right\rangle & 0 \\
0 & -\left\langle n_{\uparrow}\right\rangle
\end{array}\right) .
\end{gathered}
$$

Comparing with

$$
\begin{aligned}
\mathbf{G}\left(\mathbf{k}, i k_{n}\right) & =\left(i k_{n} \mathbf{I}-\left(\varepsilon_{\mathbf{k}}-\mu\right) \sigma_{z}-\mathbf{\Sigma}\left(\mathbf{k}, i k_{n}\right)\right)^{-1} \\
& =\frac{1}{i k_{n}} \mathbf{I}+\frac{1}{\left(i k_{n}\right)^{2}}\left[\left(\varepsilon_{\mathbf{k}}-\mu\right) \sigma_{z}+\mathbf{\Sigma}\left(\mathbf{k}, i k_{n}\right)\right]+\ldots
\end{aligned}
$$

it is clear, using spin-rotational invariance, that

$$
\lim _{i k_{n} \rightarrow \infty} \boldsymbol{\Sigma}\left(\mathbf{k}, i k_{n}\right)=U\left\langle n_{\downarrow}\right\rangle \sigma_{z} .
$$

An expression where this asymptotic behavior is explicit may easily be obtained from the general formula for the self-energy specialized to zero external field, namely Eq.(34) with $\Theta=0$. Returning to our general discussion Eq.(23) will help understand the point we were making. In zero external field, we have

$$
\boldsymbol{\Sigma}(1,2)=U \mathbf{X}\left(1, \overline{3}, 1^{\mp}, 1^{\mp}\right) \mathbf{G}^{-1}(\overline{3}, 2)=-\left.U \frac{\delta}{\delta \boldsymbol{\Theta}\left(1^{\mp}, 1^{\mp}\right)} \mathbf{G}(1, \overline{3} ;\{\boldsymbol{\Theta}\})\right|_{\Theta=0} \mathbf{G}^{-1}(\overline{3}, 2)
$$

or, looking only at the non-vanishing elements,

$$
\begin{aligned}
& \Sigma_{11}(1,2)=-\left.U \frac{\delta G_{21}\left(1^{+}, \overline{3} ;\{\boldsymbol{\Theta}\}\right)}{\delta \theta^{*}(1,1)}\right|_{\boldsymbol{\Theta}=0} G_{11}^{-1}(\overline{3}, 2) \\
& \Sigma_{22}(1,2)=-\left.U \frac{\delta G_{12}(1, \overline{3} ;\{\boldsymbol{\Theta}\})}{\delta \theta\left(1^{+}, 1^{+}\right)}\right|_{\boldsymbol{\Theta}=0} G_{22}^{-1}(\overline{3}, 2)
\end{aligned}
$$

Substituting the Bethe-Salpeter equations (46)(47) in the last two expressions we have the equivalent expressions, 


$$
\begin{aligned}
& \Sigma_{11}(1,2)=-U G_{22}\left(1^{+}, 1\right) \delta(1-2)-\left.\left.U G_{22}\left(1^{+}, \overline{4}\right) \frac{\delta \Sigma_{21}(\overline{4}, 2 ;\{\boldsymbol{\Theta}\})}{\delta G_{21}(\overline{6}, \overline{7})}\right|_{\boldsymbol{\Theta}=\mathbf{0}} \frac{\delta G_{21}(\overline{6}, \overline{7} ;\{\boldsymbol{\Theta}\})}{\delta \theta^{*}(1,1)}\right|_{\boldsymbol{\Theta = 0}} \\
& \Sigma_{22}(1,2)=-U G_{11}\left(1,1^{+}\right) \delta\left(1^{+}-2\right)-\left.\left.U G_{11}(1, \overline{4}) \frac{\delta \Sigma_{12}(\overline{4}, 2 ;\{\boldsymbol{\Theta}\})}{\delta G_{12}(\overline{6}, \overline{7})}\right|_{\boldsymbol{\Theta}=\mathbf{0}} \frac{\delta G_{12}(\overline{6}, \overline{7} ;\{\boldsymbol{\Theta}\})}{\delta \theta\left(1^{+}, 1^{+}\right)}\right|_{\boldsymbol{\Theta}=\mathbf{0}} .
\end{aligned}
$$

One may easily check that the terms proportional to $\delta(1-2)$ in these two exact expressions are precisely the HartreeFock contribution, Eq.(54).

The skeleton diagram representation of the Bethe-Salpeter equation (46) and of the self-energy Eq.(58) appear in Fig.1 of the accompanying paper. This skeleton diagram representation is not necessary to understand the rest of the paper but may be useful for physical understanding. The non-perturbative approach developed in the following section does not directly correspond to the summation of an infinite subset of diagrams.

\section{A SYSTEMATIC NON-PERTURBATIVE APPROACH}

A brief reminder of how Hartree-Fock theory is derived in the functional derivative approach will motivate the twoparticle self-consistent approach. At this first level of approximation (TPSC), one has $\mathbf{G}^{(1)}, \boldsymbol{\Sigma}^{(1)}$ in the presence of $\boldsymbol{\Theta}$ and the corresponding irreducible vertices and susceptibilities are obtained from the functional derivative approach. The only unknown quantity, double occupancy $\left\langle n_{\uparrow} n_{\downarrow}\right\rangle$, may be obtained self-consistently by using what we will call the local-pair sum rule. The local-pair sum rule is a simple consequence of the fluctuation-dissipation theorem. An improved approximation for the self-energy, $\boldsymbol{\Sigma}^{(2)}$, will be found in section III D. We conclude with a discussion of an internal accuracy check that, as in the repulsive case, helps delineate the domain of validity of the approach.

\section{A. A perspective: Conserving approximations and Hartree-Fock theory}

In conserving approximations, the self-energy is obtained from a functional derivative of the Luttinger-Ward functional [19] that is computed from skeleton diagrams. Irreducible vertices for response functions are then obtained from appropriate functional derivatives. The Hartree-Fock approach is a special case of conserving approximation. The more standard way to derive the Hartree-Fock approach is to treat the general equation for the self-energy Eq.(21) in the presence of the auxiliary field as if Wick's theorem applied to the right-hand side of that equation. More specifically, Eq.(21) becomes, in the Hartree-Fock approximation

$$
\boldsymbol{\Sigma}^{H F}(1, \overline{3} ;\{\boldsymbol{\Theta}\}) \mathbf{G}^{H F}(\overline{3}, 2 ;\{\boldsymbol{\Theta}\})=U\left(\begin{array}{cc}
-G_{22}^{H F}\left(1^{+}, 1 ;\{\boldsymbol{\Theta}\}\right) & G_{12}^{H F}(1,1 ;\{\boldsymbol{\Theta}\}) \\
G_{21}^{H F}(1,1 ;\{\boldsymbol{\Theta}\}) & -G_{11}^{H F}\left(1,1^{+} ;\{\boldsymbol{\Theta}\}\right)
\end{array}\right) \mathbf{G}^{H F}(1,2 ;\{\boldsymbol{\Theta}\})
$$

or

$$
\boldsymbol{\Sigma}^{H F}(1,2 ;\{\boldsymbol{\Theta}\})=U\left(\begin{array}{cc}
-G_{22}^{H F}\left(1^{+}, 1 ;\{\boldsymbol{\Theta}\}\right) & G_{12}^{H F}(1,1 ;\{\boldsymbol{\Theta}\}) \\
G_{21}^{H F}(1,1 ;\{\boldsymbol{\Theta}\}) & -G_{11}^{H F}\left(1,1^{+} ;\{\boldsymbol{\Theta}\}\right)
\end{array}\right) \delta(1-2)
$$

The corresponding irreducible vertices for the Bethe-Salpeter equation governing the pair fluctuations, Eq.(43) are

$$
\left.\frac{\delta \Sigma_{12}^{H F}(1,2 ;\{\boldsymbol{\Theta}\})}{\delta G_{12}^{H F}(3,4 ;\{\boldsymbol{\Theta}\})}\right|_{\boldsymbol{\Theta}=\mathbf{0}}=\left.\frac{\delta \Sigma_{21}^{H F}(1,2 ;\{\boldsymbol{\Theta}\})}{\delta G_{21}^{H F}(3,4 ;\{\boldsymbol{\Theta}\})}\right|_{\boldsymbol{\Theta}=\mathbf{0}}=U \delta(1-2) \delta(1-3) \delta(1-4)
$$

which leads to the simplest T-matrix type approximation. In the final calculations, we only need the self-energy in zero field. There it is purely diagonal and given by

$$
\boldsymbol{\Sigma}^{H F}(1,2)=\sigma_{z} U\left\langle n_{\downarrow}\right\rangle \delta(1-2)
$$

Returning momentarily to the original equation that was approximated, Eq.(21), it is important to realize that the Hartree-Fock approximation satisfies an exact result. First note that the four point function becomes simple when point 2 becomes the same as 1 . In that case, this quantity takes a simple form that, because of the anticommutation relations, depends on whether $2 \rightarrow 1^{+}$or $2 \rightarrow 1^{-}$. More specifically, we have the exact results 


$$
\begin{gathered}
\boldsymbol{\Sigma}(1, \overline{3} ;\{\boldsymbol{\Theta}\}) \mathbf{G}\left(\overline{3}, 1^{+} ;\{\boldsymbol{\Theta}\}\right)=U\left(\begin{array}{cc}
\left\langle n_{\uparrow}(1) n_{\downarrow}(1)\right\rangle_{\boldsymbol{\Theta}} & G_{12}(1,1 ;\{\boldsymbol{\Theta}\}) \\
0 & \left\langle n_{\uparrow}(1)\left(n_{\downarrow}(1)-1\right)\right\rangle_{\boldsymbol{\Theta}}
\end{array}\right) \\
\boldsymbol{\Sigma}(1, \overline{3} ;\{\boldsymbol{\Theta}\}) \mathbf{G}\left(\overline{3}, 1^{-} ;\{\boldsymbol{\Theta}\}\right)=U\left(\begin{array}{cc}
\left\langle n_{\downarrow}(1)\left(n_{\uparrow}(1)-1\right)\right\rangle_{\boldsymbol{\Theta}} & 0 \\
-G_{21}(1,1 ;\{\boldsymbol{\Theta}\}) & \left\langle n_{\uparrow}(1) n_{\downarrow}(1)\right\rangle_{\boldsymbol{\Theta}}
\end{array}\right) .
\end{gathered}
$$

In zero field, the difference of these two exact results is

$$
\boldsymbol{\Sigma}(1, \overline{3}) \mathbf{G}\left(\overline{3}, 1^{+}\right)-\boldsymbol{\Sigma}(1, \overline{3}) \mathbf{G}\left(\overline{3}, 1^{-}\right)=\sigma_{z} U\left\langle n_{\downarrow}\right\rangle .
$$

Given what we have said in section II E about the exact high-frequency behavior of the self-energy, the reader will not be surprised to learn that the Hartree-Fock approximation does satisfy the relation Eq.(66). Indeed, this exact relation is sensitive only to the high-frequency limit of the self-energy, as may be proven by writing down explicitly the 1,1 component of Eq.(66) in Fourier-Matsubara space as follows

$$
\begin{gathered}
+\frac{T}{N} \sum_{\mathbf{k}} \sum_{i k_{n}}\left(\frac{\Sigma\left(\mathbf{k}, i k_{n}\right)}{i k_{n}-\left(\varepsilon_{\mathbf{k}}-\mu\right)-\Sigma\left(\mathbf{k}, i k_{n}\right)}-\frac{U\left\langle n_{\downarrow}\right\rangle}{i k_{n}}\right)\left(e^{-i k_{n} 0^{-}}-e^{-i k_{n} 0^{+}}\right) \\
\frac{T}{N} \sum_{\mathbf{k}} \sum_{i k_{n}}\left[\frac{U\left\langle n_{\downarrow}\right\rangle}{i k_{n}}\left(e^{-i k_{n} 0^{-}}-e^{-i k_{n} 0^{+}}\right)\right]=U\left\langle n_{\downarrow}\right\rangle
\end{gathered}
$$

In this expression, the first sum vanishes because we have added and subtracted a term that makes it convergent without the need for convergence factors $e^{-i k_{n} 0^{ \pm}}$. Hence, only the last sum survives. The result is a direct manifestation of the anticommutation relations in the asymptotic behavior of the Green function.

\section{B. Two-particle Self-Consistency and irreducible vertex}

We will call the two exact results Eqs.(64) and (65) $\operatorname{Tr}[\Sigma G]$ for short cut. They are simply related to the potential energy (and hence to double occupancy), a crucial quantity for the Hubbard Hamiltonian. Furthermore, they can be considered as initial conditions for the true expression that defines the self-energy in the most general case, $\boldsymbol{\Sigma}(1, \overline{3} ;\{\boldsymbol{\Theta}\}) \mathbf{G}(\overline{3}, 2 ;\{\boldsymbol{\Theta}\})$ where point 2 has moved away from point one. Hence, in the first step of the approximation that we propose, we perform, as in conserving approximations, a Hartree-Fock like factorization in an external field, but we add the constraint that the factorization becomes exact when $2 \rightarrow 1^{+}$or $2 \rightarrow 1^{-}$. More specifically, in analogy with the factorization Eq.(60) we start from the ansatz

$$
\boldsymbol{\Sigma}^{(1)}(1, \overline{3} ;\{\boldsymbol{\Theta}\}) \mathbf{G}^{(1)}(\overline{3}, 2 ;\{\boldsymbol{\Theta}\})=U\left(\begin{array}{cc}
-G_{22}^{(1)}\left(1^{+}, 1 ;\{\boldsymbol{\Theta}\}\right) & G_{12}^{(1)}(1,1 ;\{\boldsymbol{\Theta}\}) \\
G_{21}^{(1)}(1,1 ;\{\boldsymbol{\Theta}\}) & -G_{11}^{(1)}\left(1,1^{+} ;\{\boldsymbol{\Theta}\}\right)
\end{array}\right) \mathbf{A}(\{\boldsymbol{\Theta}\}) \mathbf{G}^{(1)}(1,2 ;\{\boldsymbol{\Theta}\})
$$

where the matrix $\mathbf{A}(\{\boldsymbol{\Theta}\})$ is obtained by requiring that the exact relations Eqs.(64) and (65) be satisfied [20]. Setting alternatively $2 \rightarrow 1^{+}$or $2 \rightarrow 1^{-}$in the last expression, and requiring equality with the respective exact $\operatorname{Tr}[\Sigma G]$ expression, we find, using spin rotational invariance, that in either case there is a unique solution for the off diagonal elements

$$
\boldsymbol{\Sigma}_{12}^{(1)}(1,2 ;\{\boldsymbol{\Theta}\})=U \frac{\left\langle n_{\uparrow}\left(1-n_{\downarrow}\right)\right\rangle_{\boldsymbol{\Theta}} G_{12}^{(1)}(1,1 ;\{\boldsymbol{\Theta}\}) \delta(1-2)}{\left\langle n_{\uparrow}\right\rangle_{\boldsymbol{\Theta}}\left\langle 1-n_{\downarrow}\right\rangle_{\boldsymbol{\Theta}}-G_{12}^{(1)}(1,1 ;\{\boldsymbol{\Theta}\}) G_{21}^{(1)}(1,1 ;\{\boldsymbol{\Theta}\})}
$$

with the analogous expression when the Nambu matrix indices 1 and 2 are inverted. It should be clear from this result that the functional dependence of $\mathbf{A}(\{\boldsymbol{\Theta}\})$ on external field is only through $\mathbf{G}^{(1)}\left(1,1^{ \pm} ;\{\boldsymbol{\Theta}\}\right)$ and double occupancy $\left\langle n_{\downarrow} n_{\uparrow}\right\rangle_{\Theta}$. Nevertheless, this establishes a strong self-consistency relation between one and two-particle quantities that is absent from any standard diagrammatic approach. That is why we called this portion of the approach "Two Particle Self-Consistent" (TPSC) [11]. It is important also to note that the superscript (1) refers to what, in earlier publications, [12] we called the zeroth step of the approximation. As we will see in a moment, at this level of approximation the diagonal part of $\boldsymbol{\Sigma}^{(1)}$ is a constant when $\boldsymbol{\Theta}=\mathbf{0}$. Hence, in this limit, $\mathbf{G}^{(1)}$ is equal to a bare propagator once the chemical potential is adjusted to obtain the proper filling. That is why we had referred to this level of approximation with superscript (0) in earlier publications. It is important to notice however that $\boldsymbol{\Sigma}^{(1)}$ (or 
$\boldsymbol{\Sigma}^{(0)}$ in the former notation) may have a dependence on external magnetic field, for example, that is absent in the non-interacting case.

The particle-particle irreducible vertices appearing in the Bethe-Salpeter Eqs.(47) and (46) are obtained from functional differentiation of the self-energy Eq.(70a) as in any conserving approximation. Since when $\Theta=0$ all off diagonal function such as $G_{12}^{(1)}(1,1), G_{21}^{(1)}(1,1)$ or $\delta\left\langle n_{\downarrow}\left(1-n_{\uparrow}\right)\right\rangle_{\Theta} / \delta G_{12}^{(1)}(1,1 ;\{\boldsymbol{\Theta}\})$ and the like vanish, we are left with

$$
\begin{aligned}
\left.\frac{\delta \Sigma_{12}^{(1)}(1,2 ;\{\boldsymbol{\Theta}\})}{\delta G_{12}^{(1)}(3,4 ;\{\boldsymbol{\Theta}\})}\right|_{\boldsymbol{\Theta}=\mathbf{0}} & =\left.\frac{\delta \Sigma_{21}^{(1)}(1,2 ;\{\boldsymbol{\Theta}\})}{\delta G_{21}^{(1)}(3,4 ;\{\boldsymbol{\Theta}\})}\right|_{\boldsymbol{\Theta}=\mathbf{0}}=U \frac{\left\langle n_{\downarrow}\left(1-n_{\uparrow}\right)\right\rangle}{\left\langle n_{\downarrow}\right\rangle\left\langle 1-n_{\uparrow}\right\rangle} \delta(1-2) \delta(1-3) \delta(1-4) \\
& \equiv U_{p p} \delta(1-2) \delta(1-3) \delta(1-4) .
\end{aligned}
$$

The irreducible vertex $U_{p p}$ is still local, as in the Hartree-Fock approximation, but, contrary to Hartree-Fock, the bare vertex is dressed. The function that dresses the vertex is simply related to double occupancy. It can be determined from the Bethe-Salpeter equation itself by using the fluctuation-dissipation theorem, allowing us to close the system of equations.

\section{An approximate expression for $\Sigma^{(1)}$ in the TPSC approach}

The self-energy entering $\mathbf{G}^{(1)}$ in zero external field is diagonal. There is however an apparent paradox. Indeed, substituting the TPSC factorization Eq.(69) on the left-hand side of the two $\operatorname{Tr}[\Sigma G]$ equation Eqs.(64) and (65), all for $\boldsymbol{\Theta}=\mathbf{0}$, seems to give two different solutions for the diagonal value of $\mathbf{A}(\{\boldsymbol{\Theta}\})$, and hence for the corresponding $\boldsymbol{\Sigma}^{(1)}$. Let us use $\boldsymbol{\Sigma}^{(1)}$ with a + or - index depending on whether they are the solution of Eqs.(64) or (65). In either case, $\boldsymbol{\Sigma}^{(1)}(1-2)$ is proportional to $\delta(1-2)$, so that using $G_{11 \pm}^{(1)}\left(1,1^{+}\right)=\left\langle n_{\uparrow}(1)\right\rangle$ and $G_{11 \pm}^{(1)}\left(1,1^{-}\right)=-1+\left\langle n_{\uparrow}(1)\right\rangle$ and the like for $G_{22}^{(1)}$, one finds,

$$
\begin{gathered}
\Sigma_{11+}^{(1)}(1,2)=-\Sigma_{22-}^{(1)}(1,2)=\left(U-U_{p p}\left\langle 1-n_{\downarrow}\right\rangle\right) \delta(1-2) \\
\Sigma_{11-}^{(1)}(1,2)=-\Sigma_{22+}^{(1)}(1,2)=U_{p p}\left\langle n_{\uparrow}\right\rangle \delta(1-2)
\end{gathered}
$$

From these results, keeping the filling fixed we have

$$
\boldsymbol{\Sigma}_{+}^{(1)}(1, \overline{3}) G_{+}^{(1)}\left(\overline{3}, 1^{+}\right)-\boldsymbol{\Sigma}_{-}^{(1)}(1, \overline{3}) \mathbf{G}_{-}^{(1)}\left(\overline{3}, 1^{-}\right)=\sigma_{z} U\left\langle n_{\uparrow}(1)\right\rangle
$$

This suggests that the antisymmetric combination $\boldsymbol{\Sigma}_{+}^{(1)}-\boldsymbol{\Sigma}_{+}^{(1)}$ is related to the high-frequency asymptotic behavior of the self-energy, as in the Hartree-Fock case. The symmetric combination on the other hand should not depend on convergence factors, as can be seen by using arguments analogous to those of Eq.(67). This quantity should then be a measure of the low-frequency behavior of the self-energy. With $n \equiv\left\langle n_{\downarrow}\right\rangle+\left\langle n_{\uparrow}\right\rangle$ the quantity

$$
\frac{1}{2}\left(\boldsymbol{\Sigma}_{+}^{(1)}(1,2)+\boldsymbol{\Sigma}_{-}^{(1)}(1,2)\right)=\sigma_{z}\left(\frac{U}{2}-\frac{U_{p p}(1-n)}{2}\right) \delta(1-2)
$$

we expect, plays the role of a chemical potential shift, $\mu^{(1)}-\mu_{0}$, with respect to the non-interacting value $\mu_{0}$. We use the notation $\mu^{(1)}$ with a superscript (1) to note that this is the chemical potential corresponding to the self-energy $\Sigma^{(1)}$. We will give additional supporting evidence for this conjecture in section IV that discusses exact results satisfied by our approach and in Appendix A. In actual calculations the chemical potential used in the Green function occurring at this step of the calculation is determined from the condition $G_{11}^{(1)}\left(1,1^{+}\right)=\left\langle n_{\uparrow}(1)\right\rangle$ with

$$
G_{11}^{(1)}\left(\mathbf{k}, i k_{n}\right)=\frac{1}{i k_{n}-\varepsilon_{\mathbf{k}}+\mu^{(1)}-\mathbf{\Sigma}_{11}^{(1)}\left(\mathbf{k}, i k_{n}\right)}
$$

$\boldsymbol{\Sigma}_{11}^{(1)}\left(\mathbf{k}, i k_{n}\right)$ in the combination $\mu^{(1)}-\boldsymbol{\Sigma}_{11}^{(1)}\left(\mathbf{k}, i k_{n}\right)$ is a constant so that $\mu^{(1)}-\boldsymbol{\Sigma}_{11}^{(1)}\left(\mathbf{k}, i k_{n}\right)$ equals the non-interacting chemical potential $\mu_{0}$.

It is important to keep in mind the following. The approximate analytical expression Eq.(76) for $\boldsymbol{\Sigma}^{(1)}$ and the corresponding chemical potential $\mu^{(1)}$ are useful for analytical arguments and numerical estimates [21]. However, more fundamentally, the chemical potential is a thermodynamic quantity that can be computed in a consistent way, along with all other thermodynamic quantities. In diagrammatic methods, the Luttinger-Ward functional provides a systematic method to obtain such thermodynamically consistent results. [19] Since our approach is non-perturbative, another approach must be used. At this point, $\mu^{(1)}$ is a first estimate for the true chemical potential. A better estimate is $\mu^{(2)}$, corresponding to $\boldsymbol{\Sigma}^{(2)}$, the next level of approximation for the self-energy. 


\section{TPSC plus improved approximation for the self-energy}

Let us summarize what we have up to now. In this TPSC approach for the attractive Hubbard model, there is only one particle-particle irreducible vertex $U_{p p}$ Eq.(71). In addition, the two pair susceptibilities that we need, namely

$$
\begin{aligned}
& -\left.\frac{\delta G_{21}(2,2 ;\{\boldsymbol{\Theta}\})}{\delta \theta^{*}(1,1)}\right|_{\boldsymbol{\Theta}=\mathbf{0}}=-\left\langle T_{\tau}\left[\psi_{\downarrow}(1) \psi_{\uparrow}(1) \psi_{\downarrow}^{\dagger}(2) \psi_{\uparrow}^{\dagger}(2)\right]\right\rangle \\
& -\left.\frac{\delta G_{12}(2,2 ;\{\boldsymbol{\Theta}\})}{\delta \theta(1,1)}\right|_{\mathbf{\Theta}=\mathbf{0}}=-\left\langle T_{\tau}\left[\psi_{\uparrow}^{\dagger}(1) \psi_{\downarrow}^{\dagger}(1) \psi_{\uparrow}(2) \psi_{\downarrow}(2)\right]\right\rangle
\end{aligned}
$$

are quite easily related by the operation $2 \leftrightarrow 1$, and anticommutation $\psi_{\uparrow}(2) \psi_{\downarrow}(2)=-\psi_{\downarrow}(2) \psi_{\uparrow}(2)$. So we define

$$
\chi_{p}(1,2) \equiv-\left.\frac{\delta G_{21}(2,2 ;\{\boldsymbol{\Theta}\})}{\delta \theta^{*}(1,1)}\right|_{\boldsymbol{\Theta}=\mathbf{0}}=-\left.\frac{\delta G_{12}(1,1 ;\{\boldsymbol{\Theta}\})}{\delta \theta(2,2)}\right|_{\boldsymbol{\Theta}=\mathbf{0}} .
$$

The equality of these two functions is a reflection of the fact that for on-site pairing, the Pauli principle makes the triplet channel vanish. Hence, substituting the particle-particle irreducible vertex Eq.(71) in one of the Bethe-Salpeter equations Eq.(47) and expressing the result in terms of the ordinary Matsubara Green functions

$$
\begin{aligned}
& G_{\uparrow}^{(1)}(1,2)=G_{11}^{(1)}(1,2) \\
& G_{\downarrow}^{(1)}(1,2)=-G_{22}^{(1)}(2,1)
\end{aligned}
$$

we find the pair susceptibility at the first level of approximation $\chi_{p}^{(1)}(1,2)$,

$$
\chi_{p}^{(1)}(1,2)=G_{\uparrow}^{(1)}(1,2) G_{\downarrow}^{(1)}(1,2)-U_{p p} G_{\uparrow}^{(1)}(1, \overline{4}) \chi_{p}^{(1)}(\overline{4}, 2) G_{\downarrow}^{(1)}(1, \overline{4}) .
$$

In Fourier space and with the definitions, $q=\left(\mathbf{q}, i q_{n}\right), q_{n}=2 n \pi T, k=\left(\mathbf{k}, i k_{n}\right), k_{n}=(2 n+1) \pi T$ and

$$
\chi_{i r}^{(1)}(q)=\frac{T}{N} \sum_{k} G_{\downarrow}^{(1)}(k+q) G_{\uparrow}^{(1)}(-k)
$$

for the particle-particle susceptibility that is irreducible with respect to $U_{p p}$, the above equation will take the form

$$
\chi_{p}^{(1)}(q)=\frac{\chi_{i r}^{(1)}(q)}{1+U_{p p} \chi_{i r}^{(1)}(q)}
$$

To close the set of equations, we need to solve for the irreducible vertex

$$
U_{p p}=U \frac{\left\langle n_{\downarrow}\left(1-n_{\uparrow}\right)\right\rangle}{\left\langle n_{\downarrow}\right\rangle\left\langle 1-n_{\uparrow}\right\rangle} .
$$

That may be done by using either of the two exact limits $2 \rightarrow 1^{+}$and $2 \rightarrow 1^{-}$of Eqs.(78)(80)

$$
\begin{gathered}
\chi_{p}^{(1)}\left(1,1^{+}\right)=\left\langle n_{\downarrow} n_{\uparrow}\right\rangle \\
\chi_{p}^{(1)}\left(1,1^{-}\right)=1-n+\left\langle n_{\downarrow} n_{\uparrow}\right\rangle
\end{gathered}
$$

The left hand side of the latter two equations, that one could call local-pair sum rules, can be transformed into the following two sum rules when our approximation is used for the susceptibility

$$
\begin{gathered}
\chi_{p}^{(1)}\left(1,1^{+}\right)=\left\langle n_{\downarrow} n_{\uparrow}\right\rangle=\frac{T}{N} \sum_{q} \frac{\chi_{i r}^{(1)}(q)}{1+U_{p p} \chi_{i r}^{(1)}(q)} e^{-i q_{n} 0^{-}} \\
\chi_{p}^{(1)}\left(1,1^{-}\right)=1-n+\left\langle n_{\downarrow} n_{\uparrow}\right\rangle=\frac{T}{N} \sum_{q} \frac{\chi_{i r}^{(1)}(q)}{1+U_{p p} \chi_{i r}^{(1)}(q)} e^{-i q_{n} 0^{+}}
\end{gathered}
$$


We will discuss in section IV why, in our approach, both sum rules are consistent and so give exactly the same result for $U_{p p}$. Monte Carlo simulations confirm that the pair susceptibilities calculated with Eqs.(86), (89),(84) and (77) are excellent approximations from weak to intermediate coupling.

As we saw above, at this stage the self-energy $\Sigma^{(1)}$ entering the calculation is a constant determined in such a way that we have the proper filling. At high-frequency the exact limit of the self-energy is the Hartree-Fock result and the corresponding irreducible vertex is the bare interaction. In our section on exact results, we have found an expression for the self-energy, Eqs.(58)(59), that is the sum of two terms, a first one which is the highfrequency Hartree-Fock behavior and a second one that only involves the low frequency behavior of Green functions, of irreducible vertices and of susceptibilities. As we have seen above, our approximate expressions for these quantities are consistent and are low-frequency approximations. Hence, we substitute in Eqs.(58)(59) the result Eq.(83) for the susceptibility $-\delta G_{21}^{(1)}(2,2 ;\{\boldsymbol{\Theta}\}) /\left.\delta \theta^{*}(1,1)\right|_{\boldsymbol{\Theta}=\mathbf{0}}=\chi_{p}^{(1)}(1,2)$, and the corresponding results Eq.(71) for the irreducible $\operatorname{vertex} \delta \Sigma_{21}^{(1)}(1,2 ;\{\boldsymbol{\Theta}\}) /\left.\delta G_{21}^{(1)}(3,4 ;\{\boldsymbol{\Theta}\})\right|_{\boldsymbol{\Theta}=\mathbf{0}}$ and Green function $\mathbf{G}^{(1)}$. One is left with

$$
\boldsymbol{\Sigma}_{\uparrow}^{(2)}(1,2)=U G_{\downarrow}^{(1)}\left(1,1^{+}\right) \delta(1-2)-U U_{p p} G_{\downarrow}^{(1)}(2,1) \chi_{p}^{(1)}(1,2)
$$

Appendix B 1 discusses the relation to the corresponding formula in the repulsive case. Note that all the terms on the right-hand side of this equation, including the irreducible vertex $U_{p p}$, are at the same level of approximation. In particular, the irreducible vertex $U_{p p}$ that appears explicitly, is the functional derivative of the field-dependent $\boldsymbol{\Sigma}^{(1)}$ that enters $G^{(1)}$ and $\chi_{p}^{(1)}$. This is crucial for the quality of the approximation [12,22]. This type of consistency is absent in many modern self-consistent treatments whose self-energy contains renormalized Green's functions but with only bare vertices. [5] Going to Fourier space and using the full expression for the susceptibility Eq.(85), we have

$$
\boldsymbol{\Sigma}_{\uparrow}^{(2)}(k)=U n_{\downarrow}-U \frac{T}{N} \sum_{q} U_{p p} G_{\downarrow}^{(1)}(-k+q) \frac{\chi_{i r}^{(1)}(q)}{1+U_{p p} \chi_{i r}^{(1)}(q)}
$$

Spin rotational invariance gives us the result for down spins. Note that one of the vertices is bare while the other is dressed, contrary to the case where there is a Migdal theorem.

The superscript (2) on the last expression for the self-energy indicates that it is the next level of approximation. To improve the susceptibility calculation we would need the irreducible vertices corresponding to $\boldsymbol{\Sigma}_{12}^{(2)}(1,2 ;\{\boldsymbol{\Theta}\})$ which we do not have. Hence the calculation stops at this level. Physically, the collective modes are less sensitive to details of the quasiparticles, so they can be computed first with simple Green functions. The self-energy on the other hand is sensitive to the collective modes (they have zero-frequency Matsubara contributions contrary to fermionic quantities) and hence we have to take these modes into account when we want a better approximation for the self-energy.

\section{E. Internal accuracy check}

Either by returning to the derivation Eq.(56) of $\boldsymbol{\Sigma}_{\uparrow}^{(2)}(1,2)$, which involves a four point function Eq.(78) related to the pair susceptibility Eqs.(83,80) and double-occupancy Eq.(87), or by starting from the Fourier space expression Eq.(92) and using the local pair sum rule Eq.(89) for $\chi_{p}^{(1)}$ and the corresponding sum rule for $\chi_{i r}^{(1)}$, one finds that Eq.(91) satisfies

$$
\Sigma_{\uparrow}^{(2)}(1, \overline{2}) G_{\uparrow}^{(1)}\left(\overline{2}, 1^{+}\right)=\frac{T}{N} \sum_{k} \Sigma_{\uparrow}^{(2)}(k) G_{\uparrow}^{(1)}(k) e^{-i k_{n} 0^{-}}=U\left\langle n_{\downarrow} n_{\uparrow}\right\rangle
$$

$U\left\langle n_{\downarrow} n_{\uparrow}\right\rangle$ entering this equation is exactly the same as that computed from the local pair sum rule Eq.(89). This result is analogous to that found in the repulsive model. Note that $G_{\uparrow}^{(1)}$ enters the above equation. An indication of the accuracy of our approximations may be obtained by checking by how much $\Sigma_{\uparrow}^{(2)}(1, \overline{2}) G_{\uparrow}^{(2)}\left(\overline{2}, 1^{+}\right)$differs from $U\left\langle n_{\downarrow} n_{\uparrow}\right\rangle$ obtained from the local pair sum rule. We have checked that there is at most a few percent discrepancy between both calculations, except in the pseudogap regime. Note that the chemical potential $\mu^{(2)}$ entering $G_{\uparrow}^{(2)}$ must be obtained from the number conservation equation. We refer to Ref. [23] for a discussion of Luttinger's theorem in this context.

In any approximation for the many-body problem, full consistency for all correlation functions is unachievable. For example, in conserving approximations $[4,5]$ one starts from diagrams for the Luttinger-Ward functional and for the 
corresponding free energy. Then a self-energy and irreducible vertices are obtained from functional differentiation. These quantities may then be used in the Bethe-Salpeter equation to obtain the pair susceptibility. From that pair susceptibility, one can compute double occupancy through the exact result Eq.(87). The latter double occupancy is in general different from the one obtained from $\Sigma(1, \overline{2}) G\left(\overline{2}, 1^{+}\right)$since it does not contain the same set of diagrams. So if the double occupancy obtained from the susceptibility Eq.(87) is integrated over coupling constant to obtain the free energy, the result will in general be different from the original free energy. Conserving approximations are not self-consistent at the two-particle level. Other criticisms of these approaches appear in Refs. [12] and [22].

\section{EXACT RESULTS SATISFIED BY OUR NON-PERTURBATIVE APPROACH}

We briefly discuss exact relations and consistency requirements that are satisfied by our approach. Details of some of the proofs may be found in Appendix A.

\section{A. Sum rules on single-particle spectral weight}

Consider first the single-particle properties. These should be calculated with $\mathbf{G}^{(2)}$ that contains the self-energy Eq.(92) and the corresponding chemical potential. One can extract the moments of the corresponding spectral weight $\mathbf{A}^{(2)}(\mathbf{k}, \omega)$ from the high-frequency expansion of $\mathbf{G}^{(2)}\left(\mathbf{k}, i k_{n}\right)$ in analogy with Eq.(50). In the self-energy $\Sigma^{(2)}\left(\mathbf{k}, i k_{n}\right)$, Eq.(92), the Hartree-Fock contribution appears explicitly so that the exact high-frequency limit Eq.(54) is satisfied. This means that the normalization and first moment of $\mathbf{A}^{(2)}(\mathbf{k}, \omega)$ satisfy the exact results Eq.(51) and Eq.(52).

\section{B. Sum rules on pair spectral weight}

Concerning two-particle properties, more specifically the pairing susceptibility, there are a number of exact results that our approach satisfies. First there are the two local-pair sum rules Eqs.(89)(90) that are a consequence of the fluctuation-dissipation theorem. The value of $U_{p p}$ obtained from either one of them is the same. This is demonstrated in Appendix A.

The other exact properties we shall consider concern the pair spectral weight. Using the definition of the pair field $\Delta$ Eq.(8) and of the pair susceptibility Eqs.(80)(78)(79) the latter may be written as

$$
\chi_{e x}(1,2)=\left\langle T_{\tau} \Delta(1) \Delta^{\dagger}(2)\right\rangle .
$$

The subscript $e x$ stresses that we are, for now, considering properties of the exact pair susceptibility. The Lehmann representation, and the periodicity in imaginary time, allow one to show that

$$
\chi_{e x}\left(\mathbf{q}, i q_{n}\right)=\int \frac{d \omega}{\pi} \frac{\chi_{e x}^{\prime \prime}(\mathbf{q}, \omega)}{\omega-i q_{n}}
$$

where the time Fourier transform of the pair spectral weight $\chi_{e x}^{\prime \prime}(\mathbf{q}, \omega)$ is defined by

$$
\chi_{e x}^{\prime \prime}(\mathbf{q}, t)=\frac{1}{2}\left\langle\left[\Delta_{\mathbf{q}}(t), \Delta_{\mathbf{q}}^{\dagger}(0)\right]\right\rangle .
$$

From the latter definition and the equations of motion Eqs.(10)(11) one can show that the quantity $\chi_{e x}^{\prime \prime}(\mathbf{q}, \omega)$ obeys the following sum-rule

$$
\int \frac{d \omega}{\pi} \chi_{e x}^{\prime \prime}(\mathbf{q}, \omega)=\left\langle\left[\Delta_{\mathbf{q}}(0), \Delta_{\mathbf{q}}^{\dagger}(0)\right]\right\rangle=1-n
$$

where $n$ is the filling that is obtained from the single-particle Green functions entering the calculation of $\chi_{e x}^{\prime \prime}(\mathbf{q}, \omega)$. We show in Appendix A that our approximate expression for the susceptibility Eq.(85) satisfies this manifestation of the Pauli principle exactly, for all wave vectors q. That is why we can use either of the two local pair sum-rules Eqs. (89)(90) to find self-consistently the value of $\left\langle n_{\uparrow} n_{\downarrow}\right\rangle$.

Proceeding to the first moment of the pair spectral weight, it is shown in Appendix A, that 


$$
\begin{aligned}
\int \frac{d \omega}{\pi} \omega \chi_{e x}^{\prime \prime}(\mathbf{q}, \omega) & =\frac{1}{N} \sum_{\mathbf{k}}\left(\varepsilon_{\mathbf{k}}+\varepsilon_{-\mathbf{k}+\mathbf{q}}-2\left(\mu-\frac{U}{2}\right)\right)\left(1-\left\langle n_{\mathbf{k} \uparrow}\right\rangle-\left\langle n_{-\mathbf{k}+\mathbf{q} \downarrow}\right\rangle\right) \\
& =\left[\frac{1}{N} \sum_{\mathbf{k}}\left(\varepsilon_{\mathbf{k}}+\varepsilon_{-\mathbf{k}+\mathbf{q}}\right)\left(1-2\left\langle n_{\mathbf{k} \uparrow}\right\rangle\right)\right]-2\left(\mu-\frac{U}{2}\right)(1-n) .
\end{aligned}
$$

Like the previous sum rule, this result is valid for all wave vectors $\mathbf{q}$. It is a generalization of the $f$-sum rule to the case of the attractive Hubbard model, a sort of off-diagonal $f$-sum rule. It is generalized Ward identity that relates two-particle quantities on the left-hand side with quantities obtained from the one-particle Green function on the right-hand side. At half-filling, $\mu-\frac{U}{2}=0$, where there is an exact canonical transformation from the attractive to the repulsive Hubbard model, the above result reduces precisely to the $f$-sum-rule for the repulsive case. Again, the above expression Eq.(99) relates a two-particle quantity, on the left-hand side, to a single particle property, on the right-hand side. Neither the pair susceptibility nor the single-particle Green function are known exactly in our approach. Nevertheless, as shown in Appendix A, when our approximation $\chi_{p}^{(1)}$ for the pair susceptibility is substituted on the left-hand side of Eq.(99), and our expression for the corresponding single-particle Green function $\mathbf{G}^{(1)}$ is substituted on the right-hand side, the equation is satisfied exactly as long as one uses

$$
\mu^{(1)}=\mu_{0}+\frac{U}{2}-\frac{U_{p p}}{2}(1-n)
$$

for the chemical potential appearing on the right-hand side. This is consistent with the fact that the quantities entering the right-hand side of this equation must pertain to the single-particle Green functions used in the calculation of $\chi_{p}^{\prime \prime}(\mathbf{q}, \omega)$ on the left-hand side. Since the chemical potential entering $G^{(1)}$ should be $\mu^{(1)}=\mu_{0}+\Sigma^{(1)}$ to obtain the correct filling, use of the approximation Eq.(76) for $\Sigma^{(1)}$ leads to the above result Eq.(100). Recall however that Eq.(76) for $\Sigma^{(1)}$ is not rigorous. Nevertheless, away from the renormalized classical regime, where the self-energy is weakly frequency dependent, the chemical potential obtained from this formula differs little from the one obtained at the second level of approximation $\Sigma^{(2)}$ or from Monte Carlo simulations in the weak to intermediate coupling regime [14]. The result Eq.(99) can also be considered as a type of consistency condition between one and two-particle quantities analogous to the relation between $\operatorname{Tr}(\Sigma G)$ and $U\left\langle n_{\downarrow} n_{\uparrow}\right\rangle$ discussed in section III E.

\section{Miscellaneous}

The Mermin-Wagner theorem is satisfied by our approach. That theorem states that classical fluctuation effects prohibit continuous symmetry breaking in two dimensions. The proof follows the steps of Appendix A.3 Ref. [12]. The Kosterlitz-Thouless-Berezinskii [24] transition on the other hand involves algebraic order and large-scale vortex structures that are absent from the present approach. This transition is thus inaccessible to us. Far from the critical point on the other hand, one can show, following the steps Ref. [25] in analogy with the repulsive case, that there is Kanamori-Brueckner (quantum-fluctuation) type screening of $U_{p p}$ which is given by the approximate formula

$$
U_{p p} \simeq \frac{U}{1-\Lambda U} \quad ; \quad \Lambda=\frac{T}{N} \frac{1}{\left\langle n_{\uparrow}\right\rangle\left\langle 1-n_{\downarrow}\right\rangle} \sum_{q}\left[\chi_{i r}^{(1)}(q)\right]^{2}
$$

Finally, it is important to notice that all calculations are done at constant density. In particular, the Green function $G^{(1)}$ entering the calculation of $\Sigma^{(2)}$ in Eq.(92) is evaluated at the same density as the final result $G^{(2)}$. This is motivated by the existence of Luttinger's theorem which states that the volume enclosed by the Fermi surface at $T=0$ depends only on density, not on interaction. It would be unphysical to iterate from $\Sigma^{(1)}$ to $\Sigma^{(2)}$ starting from a $G^{(1)}$ whose Fermi-surface associated singularities are at locations in the Brillouin zone that never intersect those of $G^{(2)}$. The constant-density constraint ensures maximum overlap. This point of view is motivated by Luttinger's approach. It is discussed further in Ref. [26]. Luttinger's theorem should be satisfied to a very good approximation in our approach, as for the repulsive model [12].

\section{CONCLUSION}

In this paper we have presented a generalization of the approach developed in Ref. [12] to the attractive Hubbard model. We first established a number of exact results that form the basis of the approximation method that we introduced in section III. The first level of approximation (Two-Particle Self-Consistent) is based on a Hartree-Fock 
like factorization ansatz for the self-energy in the presence of an external off-diagonal field, Eq.(69). That ansatz differs from the standard Hartree-Fock factorization Eq.(60) by the presence of a constant matrix $\mathbf{A}(\Theta)$ that forces the factorization ansatz to reduce to the unfactorized four-point function in the special case where the later involves only density-density correlations (double occupancy). That ansatz leads to the irreducible vertex for pair fluctuations simply through functional differentiation. The resulting irreducible vertex, given by Eq.(86), depends on doubleoccupancy, a quantity that may then be determined self-consistently using fluctuation-dissipation theorem derived sum rules Eqs.(89) and (90). Either one of these local-pair sum rules suffices to close the system of equations since they are equivalent. That exact equivalence is satisfied in our approach because the normalization sum-rule for the pair spectral weight, Eq.(97), is obeyed. This sum rule is a manifestation of the Pauli principle.

The self-energy $\boldsymbol{\Sigma}^{(1)}$ entering the single-particle Green function at that first level of approximation is constant. The value of this constant is irrelevant for the calculation of the pair susceptibility since we work at constant filling, which means $\boldsymbol{\Sigma}^{(1)}$ can be absorbed in the chemical potential. Nevertheless, we have argued that Eq.(76) should be a good approximation for the value of the constant self-energy at that first level of approximation since it follows from a consistency requirement between the ansatz Eq.(69) and the two possible values of $\operatorname{Tr}(\Sigma G)$. In addition, Eq.(76) for $\boldsymbol{\Sigma}^{(1)}$ formally allows the first-moment $\left(\int \frac{d \omega}{\pi} \omega \chi_{p}^{\prime \prime}(\mathbf{q}, \omega)\right)$ sum rule on the pair spectral weight to be satisfied (Appendix A). That sum rule is the off-diagonal generalization of the $f$ - sum rule familiar from the particle-hole channel.

The rough approximation that the self-energy is a constant suffices to obtain a good approximation for the lowfrequency pair susceptibility since collective modes do not generally depend strongly on details of the damping of the underlying fermions. Details of single-particle damping do however depend strongly on the collective modes. It is possible then to improve our approximation for the self-energy by using our results for the collective modes in an exact formula Eq.(58) for $\Sigma$ where the high-frequency limit appears explicitely. That gives us a better (cooperontype) approximation for the self-energy Eq.(92). Clearly, that approximation does not assume that Migdal's theorem is satisfied since one of the vertices is dressed. When Migdal's theorem applies, both vertices are bare. As in previous work, [12], one can use the difference between $\Sigma_{\uparrow}^{(2)}(1, \overline{2}) G_{\uparrow}^{(2)}\left(\overline{2}, 1^{+}\right)$and $U\left\langle n_{\downarrow} n_{\uparrow}\right\rangle$ as a check on the level of accuracy of approach, as discussed in Sec.IIIE. Formal comparisons with other approaches are presented in Appendix B. Note that our approach is in the $S O(N \rightarrow \infty)$ universality class, by arguments similar to those that apply to the repulsive model. [27] Hence, the Mermin-Wagner theorem is satisfied as it should, but algebraic long-range order of the Kosterlitz-Thouless type is beyond the accuracy of any microscopic theory that does not use renormalization group arguments to reach the long wavelength limit and treat the $S O(2)$ symmetry exactly.

In summary then, in its simplest form, our generalization of Ref. [12] to the attractive Hubbard model is expressed by the three simple equations, Eqs.(86),(89) and (92) plus the constant density contraint that determines the chemical potential appropriate to the level of approximation. Extensions of this approach have also been proposed. [28] Questions related to thermodynamic consistency and calculation of other response functions will be presented in a later publication. In the accompanying paper, our approach is compared in detail with results of Quantum Monte Carlo simulations. In addition to achieving quantitative agreement with simulations, this approach predicts the appearance of a pseudogap in the single-particle spectral weight when the pair fluctuations enter the renormalized classical regime. This is qualitatively different from the results obtained from self-consistent $T$-matrix approximation, or FLEX-type approaches. The role of the low space dimension, the pair correlation length and single-particle thermal de Broglie wave length, and more generally the mechanism for the opening of this pseudogap, have been discussed in detail in Ref. [12].

\section{ACKNOWLEDGMENTS}

A.-M.S.T is indebted to Y.M. Vilk for numerous invaluable discussions. We are also indebted to Bumsoo Kyung for calculations that allowed the results of this work to be compared with Monte Carlo calculations, as well as for corrections and critical reading of the manuscript. This work was partially supported by the Natural Sciences and Engineering Research Council of Canada (NSERC), by the Fonds pour la Formation de Chercheurs et l'Aide à la Recherche (FCAR) from the Québec government and by the Canadian Institute for Advanced Research.

\section{APPENDIX A: SUM RULES AND HIGH-FREQUENCY EXPANSION FOR THE PAIR SUSCEPTIBILITY}

In this appendix we give more details on the derivation of the results presented in section IV. Let us begin with the

high-frequency expansion of the pair susceptibility $\chi_{p}\left(\mathbf{q}, i q_{n}\right)$ for any approximation that has a spectral representation such as Eq.(95). From that spectral representation, one obtains, 


$$
\chi_{p}\left(\mathbf{q}, i q_{n}\right) \approx-\int \frac{d \omega}{\pi} \chi_{p}^{\prime \prime}(\mathbf{q}, \omega)\left(\frac{1}{i q_{n}}\right)-\int \frac{d \omega}{\pi} \omega \chi_{p}^{\prime \prime}(\mathbf{q}, \omega)\left(\frac{1}{i q_{n}}\right)^{2}+\ldots
$$

Let us now consider the exact susceptibility $\chi_{e x}\left(\mathbf{q}, i q_{n}\right)$. The moments of $\chi_{e x}^{\prime \prime}(\mathbf{q}, \omega)$ that appear as coefficients of the expansion in powers of $\left(i q_{n}\right)^{-1}$ may be obtained as follows. The first one follows for all values of $\mathbf{q}$ from the equal-time commutator in Eq.(97). The next coefficient, $\int \frac{d \omega}{\pi} \omega \chi_{e x}^{\prime \prime}(\mathbf{q}, \omega)$, follows from

$$
\begin{aligned}
\int \frac{d \omega}{\pi} \omega \chi_{e x}^{\prime \prime}(\mathbf{q}, \omega) & =i\left[\frac{\partial}{\partial t} \int \frac{d \omega}{\pi} e^{-i \omega t} \chi_{e x}^{\prime \prime}(\mathbf{q}, \omega)\right]_{t=0} \\
& =i\left[\left\langle\frac{\partial}{\partial t}\left[\Delta_{\mathbf{q}}(t), \Delta_{\mathbf{q}}^{\dagger}(0)\right]\right\rangle\right]_{t=0} \\
& =-\left[\left\langle\left[\frac{\partial}{\partial \tau} \Delta_{\mathbf{q}}(\tau), \Delta_{\mathbf{q}}^{\dagger}(0)\right]\right\rangle\right]_{\tau=0}
\end{aligned}
$$

The latter equal-time commutator may be computed after $\frac{\partial}{\partial \tau} \Delta_{\mathbf{q}}(\tau)$ is rewritten with the help of the equation of motion, Eq.(10), leading to the result Eq.(99).

Since our approximate expression Eq.(85) for the pair susceptibility admits a spectral representation, its moments may be obtained from the high-frequency expansion in powers of $\left(i q_{n}\right)^{-1}$, by analogy with the method in Ref. [12]. From our approximate formula Eq.(85) $\chi_{p}^{(1)}(q)^{-1}=\chi_{i r}^{(1)}(q)^{-1}+U_{p p}$ and the large $i q_{n}$ expansion of $\chi_{i r}^{(1)}(q)$ one finds that

$$
\lim _{i q_{n} \rightarrow \infty} i q_{n} \chi_{p}^{(1)}\left(\mathbf{q}, i q_{n}\right)=\lim _{i q_{n} \rightarrow \infty} i q_{n} \chi_{i r}^{(1)}\left(\mathbf{q}, i q_{n}\right)=-(1-n)
$$

in agreement with the exact result Eq.(97). Note that the large $i q_{n}$ limit of $\chi_{i r}^{(1)}\left(\mathbf{q}, i q_{n}\right)$ must be taken after the sum over fermionic frequencies in Eq.(84).

The first moment (off-diagonal $f$ sum-rule) is given by

$$
\begin{aligned}
-\int \frac{d \omega}{\pi} \omega \chi_{p}^{\prime \prime(1)}(\mathbf{q}, \omega) & =\lim _{i q_{n} \rightarrow \infty}\left[\left(i q_{n}\right)^{2} \chi_{p}^{(1)}\left(\mathbf{q}, i q_{n}\right)+i q_{n}(1-n)\right] \\
& =\lim _{i q_{n} \rightarrow \infty}\left[\left(i q_{n}\right)^{2} \chi_{i r}^{(1)}\left(\mathbf{q}, i q_{n}\right)\left(1-U_{p p} \chi_{i r}^{(1)}\left(\mathbf{q}, i q_{n}\right)\right)+i q_{n}(1-n)\right]
\end{aligned}
$$

Substituting the exact results for the high-frequency expansion of $\chi_{i r}^{(1)}\left(\mathbf{q}, i q_{n}\right)$, one obtains,

$$
\int \frac{d \omega}{\pi} \omega \chi_{p}^{\prime \prime(1)}(\mathbf{q}, \omega)=\frac{1}{N} \sum_{\mathbf{k}}\left(\varepsilon_{\mathbf{k}}+\varepsilon_{-\mathbf{k}+\mathbf{q}}\right)\left(1-2\left\langle n_{\mathbf{k} \uparrow}\right\rangle\right)-2 \mu_{0}(1-n)+U_{p p}(1-n)^{2}
$$

In this expression, $\left\langle n_{\mathbf{k} \uparrow}\right\rangle$ is computed from $G^{(1)}$, hence is a Fermi function, and $\mu_{0}=\mu^{(1)}+\Sigma^{(1)}$ enters the Green function $G^{(1)}$ from which $\chi_{i r}^{(1)}$ is computed. Since the self-energy $\Sigma^{(1)}$ entering $G^{(1)}$ is a constant, $\mu_{0}$ coincides with the non-interacting chemical potential appropriate for the filling we are considering. Comparing with the exact result Eq.(99) we see that the chemical potential at that level of approximation should be given by $\mu^{(1)}=\mu_{0}+$ $\left(U-U_{p p}(1-n)\right) / 2$ which coincides with our proposed approximation Eq.(76). Numerically, we have checked that this approximate chemical potential is quite close to the chemical potential $\mu^{(2)}$ obtained with $\Sigma^{(2)}$ and that the latter in turn is close to those obtained from Monte Carlo simulations [14], as long as we are far from the renormalized classical regime where $\boldsymbol{\Sigma}^{(2)}$ acquires a strong frequency dependence.

\section{APPENDIX B: COMPARISON WITH OTHER APPROACHES}

We first comment on the connection to the formalism for the repulsive model. This leads us then to a discussion of the widely used Self-Consistent T-matrix approximation and of another "mixed" approach that has been extensively applied recently. [29] 


\section{Mapping to the repulsive model and comparisons with Self-Consistent $T$-matrix approximation}

At half-filling, the Lieb-Mattis canonical transformation

$$
\begin{aligned}
c_{i, \uparrow} & \rightarrow c_{i, \uparrow} \\
c_{i, \downarrow} & \rightarrow e^{i \mathbf{Q} \cdot \mathbf{r}_{i}} c_{i, \downarrow}^{\dagger}
\end{aligned}
$$

with $\mathbf{Q}=(\pi, \pi)$ maps the repulsive into the attractive Hubbard model. The same canonical transformation maps spins $S$, density $\rho$ and pairing operators into each other as follows

$$
\begin{aligned}
S^{z}(\mathbf{Q}+\mathbf{q}) & \rightarrow \rho(\mathbf{Q}+\mathbf{q}) \\
S^{+}(\mathbf{Q}+\mathbf{q}) & \rightarrow-\Delta^{\dagger}(\mathbf{q}) \\
S^{-}(\mathbf{Q}+\mathbf{q}) & \rightarrow-\Delta(\mathbf{q})
\end{aligned}
$$

For a chemical potential different from $U / 2$ (half-filling), the repulsive model maps into the attractive model at halffilling but in a finite Zeeman-coupled magnetic field. The approach presented here for the attractive model would, at half-filling, translate into the transverse-channel calculation for the repulsive model [22]. In that $U>0$ case, Ref. [30] presents only the longitudinal channel calculation. The analog calculation for the attractive Hubbard model would have lead us to two irreducible vertices. One vertex would have been for the charge fluctuations. These are related to pair fluctuations by the $S O(3)$ symmetry of the model a $n=1$, which implies that the corresponding irreducible vertex there is also $U_{p p}$. The other vertex would have been for the non-singular spin-fluctuation channel. The two vertices would have appeared in a self-energy formula that would replace Eq.(91). However, at $n=1$, the best self-energy formula for the attractive Hubbard model would be obtained from the canonical transformation of that presented in Ref. [22] which preserves crossing symmetry. For problems sufficiently far away from half-filling $\left(T_{X} \ll \mu\right)$ however, the pair fluctuations suffice. [31]

In the repulsive model case, we have presented general analytical arguments [12] as well as detailed comparisons between: Monte Carlo simulations [12,22], our approach, and self-consistent Eliashberg type approaches (such as the Fluctuation-Exchange approximation). Most of our general criticism concerning self-consistent approaches in the repulsive case apply to self-consistent $T$ - matrix plus fluctuation exchange approaches in the attractive case.

More specifically, one of the key qualitative differences between our approach and self-consistent approaches is that the latter do not predict the existence of a fluctuation-induced pseudogap in the single-particle spectral weight in two dimensions. We have demonstrated at length, through comparisons with Monte Carlo simulations [22,32] and with physical arguments [12], that this is incorrect in both the repulsive and the attractive cases.

\section{The $G G^{(0)}$ approach}

An alternate approach based on computing the irreducible susceptibility with one bare and one dressed Green function has been extensively used lately [29]. More specifically, in this approach

$$
\chi_{p}=\frac{\widetilde{\chi}_{p}}{1+U \widetilde{\chi}_{p}}
$$

where

$$
\widetilde{\chi}_{p}=\frac{T}{N} \sum_{\mathbf{k}} G_{\downarrow}(\mathbf{k}) G_{\uparrow}^{0}(-\mathbf{k}+\mathbf{q})
$$

with the self-energy entering $G$ given by

$$
\begin{aligned}
\Sigma_{\downarrow}(k) & =\frac{T}{N} \sum_{\mathbf{q}} \frac{U}{1+U \widetilde{\chi}_{p}(q)} G_{\uparrow}^{0}(q-k) e^{-i\left(q_{n}-k_{n}\right) 0^{-}} \\
& =U n_{\uparrow}^{0}-U^{2} \frac{T}{N} \sum_{\mathbf{q}} \frac{\widetilde{\chi}_{p}(q)}{1+U \widetilde{\chi}_{p}(q)} G_{\uparrow}^{0}(q-k) .
\end{aligned}
$$

Since there is a single chemical potential for the two Green functions $G_{\downarrow}$ and $G_{\uparrow}^{0}$, there are two different expressions for the occupation numbers operators $\left(n_{\mathbf{k}}\right.$ and $\left.n_{\mathbf{k}}^{0}\right)$ and for the corresponding fillings, $\left(n\right.$ and $\left.n^{0}\right)$. 
A positive aspect of this approach is that it exhibits consistency between one and two particle properties in the sense that the exact result

$$
\frac{T}{N} \sum_{k} \Sigma_{\downarrow}(k) G_{\downarrow}(k) e^{-i k_{n} 0^{-}}=U\left\langle n_{\downarrow} n_{\uparrow}\right\rangle
$$

that follows from the equation of motion, is satisfied exactly by the above approximate scheme. Indeed, starting from the approximate formula Eq.(B6) for the self-energy, and using Eq.(B5) for the susceptibility, one finds that

$$
\begin{aligned}
\frac{T}{N} \sum_{k} \Sigma_{\downarrow}(k) G_{\downarrow}(k) e^{-i k_{n} 0^{-}} & =U \frac{T}{N} \sum_{\mathbf{q}} \frac{\tilde{\chi}_{p}(q)}{1+U \widetilde{\chi}_{p}(q)} e^{-i q_{n} 0^{-}} \\
& =U\left\langle\Delta^{\dagger} \Delta\right\rangle=U\left\langle n_{\uparrow} n_{\downarrow}\right\rangle
\end{aligned}
$$

One goes from the second to the last line using the fluctuation dissipation-theorem. Hence, in this approach, the double occupancy obtained from single particle quantities (namely $\Sigma_{\downarrow} G_{\downarrow}$ ) is exactly the same as that found from the pair susceptibility, a two-particle quantity.

On the negative side, the spectral weight corresponding to the susceptibility Eq.(B4) does not satisfy the sum rules on the first two moments discussed in Eqs.(99) and (97). To show this, we first need a few sum rules on the single-particle spectral weight. Following the steps in Appendix A of Ref. [12], the high-frequency expansion of $G$, with $\Sigma$ given by Eq.(B7), gives the following sum rules for the corresponding spectral weight $A(\mathbf{k}, \omega)$

$$
\begin{gathered}
\int \frac{d \omega}{2 \pi} A(\mathbf{k}, \omega)=1 \\
\int \frac{d \omega}{2 \pi} \omega A(\mathbf{k}, \omega)=\varepsilon_{\mathbf{k}}-\mu+U n_{-\sigma}^{0} .
\end{gathered}
$$

Also, the equation of motion for $G$ gives

$$
\int \frac{d \omega}{2 \pi} \omega f(\omega) A(\mathbf{k}, \omega)=\frac{1}{N} \sum_{\mathbf{k}}\left(\varepsilon_{\mathbf{k}}-\mu\right) n_{\mathbf{k}, \sigma}+U\left\langle n_{\uparrow} n_{\downarrow}\right\rangle .
$$

The above sum rules are valid for both $G$ and $G^{(0)}$. In the latter case however, we take the Fermi function for the occupation number and $U=0$ on the right-hand side of the above equations.

We are now ready to check the sum rules for $\widetilde{\chi}_{p}$. Using the spectral representation for $G$ in the expression for the susceptibility Eq.(B5),

$$
\tilde{\chi}_{p}\left(\mathbf{q}, i q_{n}\right)=\frac{T}{N} \sum_{\mathbf{k}} \sum_{i k_{n}} \int \frac{d \omega}{2 \pi} \frac{A(\mathbf{k}, \omega)}{i k_{n}-\omega} \frac{1}{-i k_{n}+i q_{n}-\left(\varepsilon_{-\mathbf{k}+\mathbf{q}}-\mu\right)}
$$

and performing the high-frequency expansion after the summation over $i k_{n}$, one obtains

$$
\lim _{i q_{n} \rightarrow \infty}\left(i q_{n} \widetilde{\chi}_{p}\left(\mathbf{q}, i q_{n}\right)\right)=-1+n_{\downarrow}+n_{\uparrow}^{0}
$$

with the help of the normalization Eq.(B11) and first moment sum rule Eq.(B12). This should be compared with the exact result $(-1+n)$ found in Eq.(A5). The difference between the two fillings $n_{\downarrow}$ and $n_{\uparrow}^{0}$ is a measure of how much this approximation violates the Pauli principle.

Pursuing the large $i q_{n}$ expansion and using spin rotational invariance and the sum rules Eqs.(B11) to (B13) on the single-particle spectral weight, one obtains for the first moment of the pair spectral weight (off-diagonal $f$-sum rule)

$$
\begin{aligned}
\int \frac{d \omega}{\pi} \omega \chi_{p}^{\prime \prime}(\mathbf{q}, \omega)= & \frac{1}{N} \sum_{\mathbf{k}}\left[\left(\varepsilon_{\mathbf{k}}+\varepsilon_{-\mathbf{k}+\mathbf{q}}-2\left(\mu-\frac{U}{2}\right)\right)\left(1-\left\langle n_{\mathbf{k}, \downarrow}\right\rangle-\left\langle n_{-\mathbf{k}+\mathbf{q}, \uparrow}^{0}\right\rangle\right)\right. \\
& \left.-U n_{\downarrow}-U\left(\left\langle n_{\uparrow} n_{\downarrow}\right\rangle-n_{\downarrow} n_{\downarrow}\right)+2 U n_{\uparrow}^{0} n_{\downarrow}\right] .
\end{aligned}
$$

Even with $n_{\mathbf{k}}=n_{\mathbf{k}}^{0}$ there are deviations from the exact result that are linear in $U$. 
[1] P.C. Martin and J. Schwinger, Phys. Rev. 115, 1342 (1959). This paper also contains numerous references to previous work.

[2] David Pines, The Many-body problem (Benjamin, Reading, 1962). A reprint volume with early references.

[3] L.P. Kadanoff and G. Baym, Quantum Statistical Mechanics, (Benjamin, Menlo Park, 1962).

[4] Gordon Baym, Phys. Rev. 127, 1391 (1962).

[5] N.E. Bickers and D.J. Scalapino, Annals of Physics, 193, 206 (1989).

[6] A.A. Abrikosov, L.P. Gorkov and I.E. Dzyaloshinski, Methods of quantum field theory in statistical physics (PrenticeHall, Englewood Cliffs, 1963); A.L. Fetter and J.D. Walecka, Quantum theory of Many-Particle Systems (McGraw-Hill, New York, 1971); G.D. Mahan, Many-Particle Physics, (Plenum, New York, 1981); A.M. Zagoskin, Quantum Theory of Many-Body Systems, Techniques and Applications (Springer, New York, 1998); S. Doniach, and E.H. Sondheimer, Green's functions for solid state physicists, (Latest edition) (Impreial College Press, London, 1998).

[7] K.S. Singwi, M.P. Tosi, R.H. Land, and A. Sjölander, Phys. Rev. 176, 589 (1968); For a review, see K. S. Singwi and M. P. Tosi, in Solid State Physics, edited by H. Ehrenreich, F. Seitz, and D. Turnbull (Academic, New York, 1981), Vol. 36, p. 177; S. Ichimaru, Rev. Mod. Phys. 54, 1017 (1982).

[8] J. Hubbard, Proc. Roy. Soc. A276, 238 (1963); M. Gutzwiller, Phys. Rev. Lett. 10, 159 (1963); J. Kanamori, Prog. Theor. Phys. 30, 275 (1963).

[9] Antoine Georges, Gabriel Kotliar, Werner Krauth and Marcelo J. Rozenberg, Rev. Mod. Phys. 68, 13 (1996).

[10] Y.M. Vilk, Liang Chen, and A.-M.S. Tremblay, Physica C 235-240, 2235 (1994); Y.M. Vilk, Liang Chen, A.-M.S. Tremblay, Phys. Rev. B 49, 13267 (1994).

[11] Y.M. Vilk and A.-M.S. Tremblay, Europhys. Lett. 33, 159 (1996); Y.M. Vilk and A.-M.S. Tremblay, J. Phys. Chem. Solids 56, 1769 (1995).

[12] Y. M. Vilk and A.-M. S. Tremblay, J. Phys. I France 7, 1309 (1997).

[13] M.R. Hedeyati, and G. Vignale, Phys. Rev. B 40, 9044 (1989).

[14] B. Kyung, S. Allen and A.-M.S. Tremblay (accompanying paper).

[15] T. Timusk, and B.W. Statt, Rep. Prog. Phys. 62, 61 (1999).

[16] H. Mayaffre, P. Wzietek, C. Lenoir, D. Jérome, and P. Batail, Europhys. Lett. 28, 205 (1994); P. Wzietek, H. Mayaffre, D. Jérome, and S. Brazovskii, J. Phys. I France 6, 2011 (1996); K. Frikach, M. Poirier, M. Castonguay, and K. D. Truong , Phys. Rev. B 61, R6491 (2000).

[17] Ref. [3], p.43

[18] As usual [3], we assume that the only dependence of $\boldsymbol{\Sigma}$ on $\Theta$ is through its functional dependence on the Green function G, Eq.(17). That $\boldsymbol{\Sigma}$ may be expressed as a function of $\mathbf{G}$ only requires the same assumptions as those underlying the existence of a Luttinger-Ward functional [19]. Note also that out of the four functions entering the matrix $\mathbf{G}$, only two in principle suffice to express the dependence on $\Theta$ since the latter has only two non-zero matrix elements.

[19] J.M. Luttinger and J.C. Ward, Phys. Rev. 118, 1417 (1960).

[20] Alternatively, it is also possible to write the Hartree-Fock like ansatz Eq.(69) in the form $\boldsymbol{\Sigma}^{(1)}(1, \overline{3} ;\{\boldsymbol{\Theta}\}) \mathbf{G}^{(1)}(\overline{3}, 2 ;\{\boldsymbol{\Theta}\})=$ $U \mathbf{B}(\{\boldsymbol{\Theta}\}) \mathbf{G}^{(1)}(1,2 ;\{\boldsymbol{\Theta}\})$ and to find $\mathbf{B}(\{\boldsymbol{\Theta}\})$ using again the requirement that the local values, $2 \rightarrow 1^{+}$or $2 \rightarrow 1^{-}$, corresponding to $\operatorname{Tr}[\boldsymbol{\Sigma} \mathbf{G}]$, in Eqs. $(64)(65)$ be obtained exactly.

[21] For $U>0$ the corresponding expression for $\Sigma^{(1)}$ is $(n / 2)\left(U+U_{s p}(1-n)\right) /(2-n)$ (Anne-Marie Daré and A.-M.S. Tremblay, unpublished).

[22] S. Moukouri, S. Allen, F. Lemay, B. Kyung, D. Poulin, Y.M. Vilk and A.-M. S. Tremblay, Phys. Rev. B 61, 7887 (2000).

[23] Ref. [12], section 3.2.2.

[24] V.L. Berezinskii, Z. Eksp. Teor. Fiz. 59, 907 (1970); J. Kosterlitz, and D. Thouless J. Phys. C6, 1181 (1973).

[25] See Sec.3.2.1. of Ref.( [12]). The factor of 2 on the right-hand side of Eq.(43) should not be there.

[26] Ref. [12], below Eq.(47) p.1322.

[27] Anne-Marie Daré, Y.M. Vilk and A.-M.S. Tremblay, Phys. Rev. B 53, 14236 (1996).

[28] Steve Allen, PhD thesis, Université de Sherbrooke, Summer 2000 (unpublished).

[29] B. Janko, J. Maly, and K. Levin, Phys. Rev. B 56, R 11407 (1997).

[30] Ref. [12], Appendix C, p.1357.

[31] $T_{X}$ denotes the crossover temperature to the renormalized classical regime. When $T_{X} \ll \mu$, the $S O(3)$ symmetry is no-longer apparent and pair fluctuations dominate.

[32] S. Allen, H. Touchette, S. Moukouri, Y.M. Vilk, A.-M. S. Tremblay, Phys. Rev. Lett., 83, 4128 (1999). 